\title{
Missing data imputation and corrected statistics for large-scale behavioral databases
}

\author{
Pierre Courrieu • Arnaud Rey
}

Published online: 18 March 2011

(C) Psychonomic Society, Inc. 2011

\begin{abstract}
This article presents a new methodology for solving problems resulting from missing data in large-scale item performance behavioral databases. Useful statistics corrected for missing data are described, and a new method of imputation for missing data is proposed. This methodology is applied to the Dutch Lexicon Project database recently published by Keuleers, Diependaele, and Brysbaert (Frontiers in Psychology, 1, 174, 2010), which allows us to conclude that this database fulfills the conditions of use of the method recently proposed by Courrieu, BrandD'Abrescia, Peereman, Spieler, and Rey (2011) for testing item performance models. Two application programs in MATLAB code are provided for the imputation of missing data in databases and for the computation of corrected statistics to test models.
\end{abstract}

Keywords Missing data imputation - Statistics corrected for missing data Item performance behavioral databases .

Model goodness of fit

An increasing number of large-scale item performance behavioral databases have been published recently, making available a large amount of shared data for building virtual experiments testing hypotheses and models. In particular, three large-scale databases providing response times (RTs) and accuracy data for thousands of words, in standard visual word recognition tasks (lexical decision or naming),

P. Courrieu $(\bowtie) \cdot$ A. Rey

Laboratoire de Psychologie Cognitive, UMR CNRS 6146,

Université de Provence, Centre Saint Charles,

Bat. 9, Case D, 3 Place Victor Hugo,

13331 Marseille cedex 3, France

e-mail: pierre.courrieu@univ-provence.fr are now available for three different languages: the English Lexicon Project (ELP; Balota et al., 2007), the French Lexicon Project (FLP: Ferrand et al., 2010), and the Dutch Lexicon Project (DLP: Keuleers et al., 2010).

Examining RT raw data in these databases, one can observe that the amount of missing data, as a result of response errors, technical failures, or outliers, is quite important. For instance, there are about $16 \%$ missing lexical decision RTs in the DLP, which are easily countable since the raw data are simply available and the DLP used a complete experimental design in which all participants (39) responded to all test words $(14,089)$, in contrast to the ELP (40,481 words) and the FLP (39,840 words), in which each of the numerous participants responded only to a subset of the whole set of test words. Nevertheless, on the basis of the published percentages of accuracy, one can estimate that the percentage of missing lexical decision RTs is about $16 \%$ for the ELP and about 9\% for the FLP. This is not visible for final users, because they usually do not use the raw data. In fact, the databases provide an average RT over all nonmissing RTs for each test word, so missing data are not actually a problem at this stage.

However, in order to test models that predict RT performance at the item level (e.g., Perry, Ziegler, \& Zorzi, 2010; Yap \& Balota, 2009), it is necessary to know the reproducible proportion of item-related variance that is available in the data. It has recently been shown that this proportion is given by a particular intraclass correlation coefficient (ICC), which is the so-called "ICC(C, k), Cases 2 and $2 \mathrm{~A}$ " coefficient, according to the nomenclature of McGraw and Wong (1996), computed on the raw data table. This method is valid provided that the considered experimental measure fulfills an additive decomposition model that is very commonly assumed (Courrieu et al., 2011; Rey, Courrieu, Schmidt-Weigand, \& Jacobs, 2009). 
Courrieu et al. proposed an efficient test, named the expected correlation validity test (ECVT), to determine the suitability of the ICC approach for any given database. However, this test is based on a Monte Carlo permutation resampling method, which is sensitive to missing data and does not correctly work when the proportion of missing data is large (say, more than 5\%). Moreover, it can be shown that missing data act, in fact, as a parasitic source of noise that harms the data consistency and, consequently, lowers the reproducible proportion of item-related variance as measured by the ICC. The ICC itself and its confidence intervals can always be computed just by using a standard analysis of variance (ANOVA) on any raw data table, and Rey and Courrieu (2010) recently published these statistics for the DLP database. However, without the ECVT, one cannot be sure that the ICC accurately measures the reproducible proportion of item-related variance.

As we noted above, the DLP used a complete experimental design, providing a 14,089 items $\times 39$ participants data table from which one can easily compute the ICC (just by removing 33 items without valid RT data). However, this is not the case for the ELP and FLP databases, where each test word was presented only to a subset of all participants. A possible solution to this problem is to build virtual participants by mixing the data provided by several real participants in such a way that each virtual participant has an observation for each item. Using raw data to do this could have catastrophic consequences if the mixed data were provided by real participants having different response characteristics, such as, for instance, their cognitive speed (Faust, Balota, Spieler, \& Ferraro, 1999). With regard to response latencies, Faust et al. showed that interindividual variability is well modeled by linear relationships. So, a simple way of removing this linear component of the interindividual variability from the data is to transform the raw data into $Z$ scores; that is, for each real participant, all his/her valid data are centered by subtracting their average and dividing by their standard deviation. This transformation is nearly unbiased, provided that one assigns with each real participant a large random sample of items. Then $Z$ scores can be mixed to build virtual participants. So, using $Z$ scores probably represents a general solution for largescale databases. However, even using (possibly mixed) $Z$ scores, there is still a problem with missing data, because, in general, the number of valid data is not the same for all items. For instance, in the DLP RT database, after 33 items with zero valid data are removed, there remain 58 items with only 1 valid datum each, 59 items with 2 valid data each, and so on, up to 3,219 items with 39 valid data each. This function is plotted in Fig. 1.

Statisticians have widely studied the problem of missing data, which is often present in large-scale studies. This has led them to develop imputation methods that allow

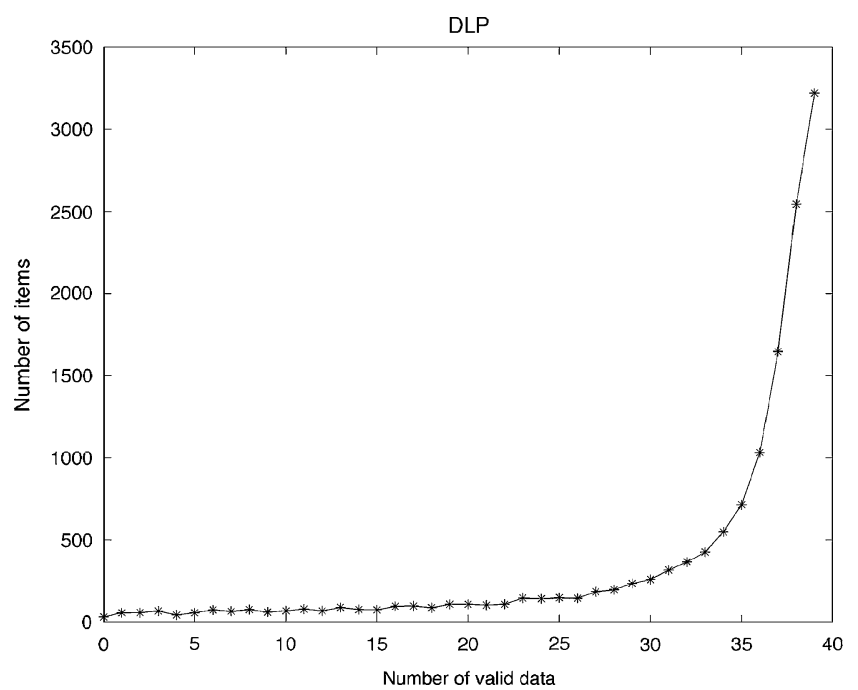

Fig. 1 Plot of the number of items as a function of the number of valid observations (response times) per item in the Dutch Lexicon Project database (Keuleers et al., 2010)

replacing missing data with suitable estimates, avoiding the introduction of statistical biases, as much as possible. Many imputation methods rely on regressionlike techniques that are not suitable to the type of data considered here. However, there is at least one imputation method that seems appropriate for our problem. This is the so-called adjusted random imputation (ARI) method (Chen, Rao, \& Sitter, 2000), which is very simple and nearly unbiased and which, in addition, preserves the original data mean values, thus avoiding modifications of the data usually provided to final users of large-scale databases.

In the next section, we describe the ARI method of Chen et al. (2000), and we rapidly examine its drawbacks for an application to the type of database considered in this article. In the third section, we show that the ICC of data tables with missing data is biased, and we describe a suitable estimate of the "true" ICC of any Z-score-type data table with missing data. In the fourth section, we describe a new missing data imputation method, called column and row adjusted random imputation (CRARI), which is a suitable extension of the ARI method that allows adjusting the data ICC. A MATLAB program implementing the CRARI method is listed in Appendix 1, together with an example of application to real data. In the fifth section, we demonstrate, using artificial data, that the ECVT (Courrieu et al., 2011) provides the same results for data tables without missing data than for similar data tables where a substantial proportion of data has been removed and imputed by the CRARI method. In the sixth section, we apply the CRARI imputation method to the DLP database, which then allows us to apply the ECVT and to conclude positively about the relevance of the ICC approach for this database. In the seventh section, we observe that missing 
Table 1 Illustration of the adjusted random imputation (ARI) method. In this example, the original data set for a given item $i$ is composed of five response times (RTs) and two missing values (from participants 3 and 6). During step 1 of the ARI method, each missing value is replaced by a randomly selected valid datum (with replacement).
During step 2, the average of these two replaced RTs (560) is subtracted from each replaced RT. During step 3, the average of the valid data is added to the previous replaced values. One can verify that the mean RT for the final set of values is equal to the mean RT for the original data set

\begin{tabular}{|c|c|c|c|c|c|c|c|c|}
\hline Participant & 1 & 2 & 3 & 4 & 5 & 6 & 7 & Means \\
\hline Item $i$ data & 500 & 570 & missing & 630 & 520 & missing & 620 & 568 \\
\hline Step 1 & 500 & 570 & $\underline{620}$ & 630 & 520 & $\underline{500}$ & 620 & $\underline{560}$ \\
\hline Step 2 & 500 & 570 & $620-\underline{560}=60$ & 630 & 520 & $500-\underline{560}=-60$ & 620 & \\
\hline Step 3 & 500 & 570 & $60+5 \overline{68}=628$ & 630 & 520 & $-60+\overline{568}=508$ & 620 & 568 \\
\hline
\end{tabular}

data not only degrade the ICC, but also degrade the average values used as item performance measures. So we propose corrected correlation statistics suitable to solve this problem when one tests models and predictors for the considered data. A MATLAB program computing these statistics is listed in Appendix 2, together with an example of application to real data. Finally, we conclude in the eighth section.

\section{Adjusted random imputation method}

The ARI method (Chen et al., 2000) allows replacing missing data, in a data table, with random estimates in such a way that the average of each row (item) remains the same as in the raw data table and the expected variance in each row is equal to the observed variance of valid raw data in the same row. Given that the empirical mean and variance are unbiased consistent estimators of the parent parameters, one can hope that the imputation is at least approximately unbiased for the mean and variance. In addition, the method is very simple. For each item, one first replaces each missing datum with a valid datum randomly selected (with replacement) from among all the valid data for the considered item. Then one subtracts the average of the replaced datum from these data, and one adds to them the average of the valid data, which provides to the imputation the properties mentioned above (see Table 1 for a concrete example).

Unfortunately, this elegant method also has some drawbacks that we must take into account. First, in cases in which there is only one valid datum for a given item, the variance of the data under imputation is zero, because only one value is used. Since it is not rare that only one valid datum is available for an item in large-scale databases (e.g., this occurs for 58 items in the DLP), there is here a possible variance bias. However, the variance in rows, by itself, is not the most important characteristic for the use of item databases. What is actually important, in this case, is the consistency of the data as measured by the ICC, so what we should obtain is an approximately unbiased ICC estimate under missing data imputation.
In order to examine the performance of the ARI method from this point of view, we built artificial data tables, of $Z$ score type, with known ICCs. Then we gradually degraded these data tables by increasing the proportion of (randomly) missing data. In each case, the $Z$ scores were recomputed on the degraded data, and the ICC of the obtained data table was computed. Then the ARI imputation method was applied to this table, and the data ICC under imputation was computed. Figure 2 shows a typical example of such an experiment, using $1,400 \times 80$ tables of $Z$ scores. As one can see in Fig. 2, the ICC of the raw data table decreases and substantially underestimates the exact ICC as the proportion of missing data increases. On the other hand, the ICC of the data table under imputation by the ARI method increases

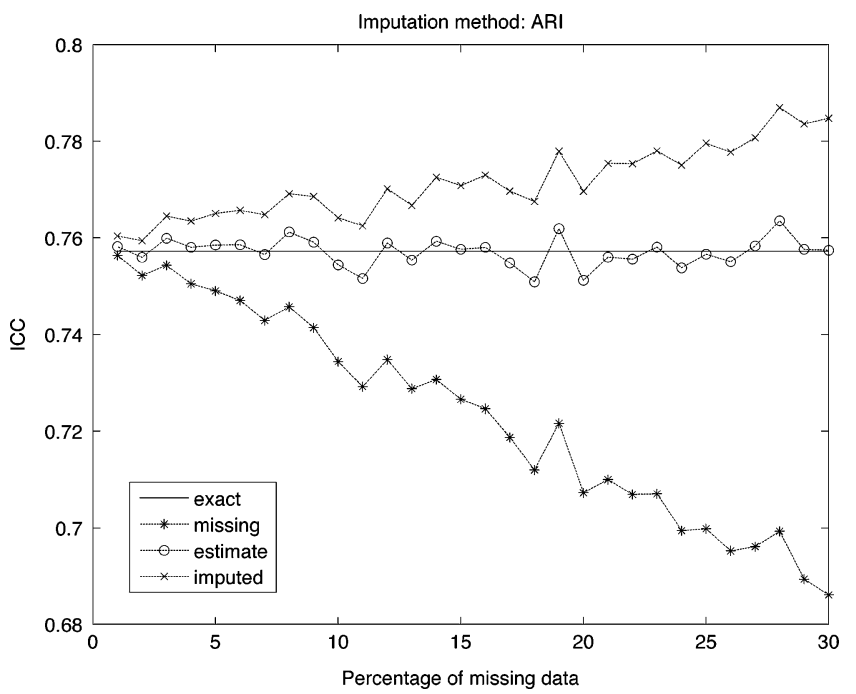

Fig. 2 Intraclass correlation coefficients (ICCs) computed from artificial 1,400 $\times 80 \mathrm{Z}$ score data tables, from an initial complete data table whose ICC was known, and gradually degraded by increasing the proportion of randomly missing data (up to $30 \%$ ). The "exact" curve corresponds to the ICC of the initial table, the "missing" curve corresponds to the ICCs of the degraded tables, and the "imputed" curve corresponds to the ICCs obtained under imputation of missing data by the adjusted random imputation (ARI) method (Chen et al., 2000). The "estimate" curve corresponds to the $\rho_{c o r}$ ICCs defined by Eq. 7 
and substantially overestimates the exact ICC as the proportion of missing data increases. Thus, neither the raw data ICC nor the ICC of data imputed by the ARI method provides a reliable estimate of the exact ICC (i.e., the ICC of the original data table before degradations). In addition, Fig. 2 shows a quite accurate estimate of the exact ICC that will be described in the next section.

In summary, the ARI method has the advantage of imputing missing data in a way that preserves the original item means. Unfortunately, this imputation method does not preserve the data consistency as measured by the ICC statistic, and it introduces consistency biases that increase as the proportion of missing data increases. So we must find another approach.

\section{Intraclass correlation coefficient corrected for missing data}

In this section, we define a suitable estimate of the exact ICC, using the ICC observed for the raw data and the proportion of missing data in the data table. Before doing this, we rapidly recall the data population model that leads to the ICC approach of the reproducible proportion of itemrelated variance (Courrieu et al., 2011; Rey et al., 2009).

Data population model Let $I$ be a population of items, let $P$ be a population of participants, and let $X$ be a behavioral measure (e.g. RT) on the $I \times P$ space, probabilized by a distribution representing, say, the probability for each pair (item, participant) to be selected in an experiment. One assumes that $X$ conforms to the usual additive decomposition model:

$X=\mu+\alpha+\beta+\varepsilon$,

where $\mu$ is the mean value of $X$ on $I \times P$, and $\alpha, \beta$, and $\varepsilon$ are three independent random variables with a mean of zero and variance $\sigma_{\alpha}^{2}, \sigma_{\beta}^{2}$, and $\sigma_{\varepsilon}^{2}$, respectively. The variable $\alpha$ is the participant effect, and it takes a constant value for each given participant. The variable $\beta$ is the item effect, and it takes a constant value for each given item. The variable $\varepsilon$ is considered as random noise; however, it can as well result from the combination of an item-participant interaction and true random noise. The variable $\beta$, whose values characterize the items, is the variable of interest in this study.

One can derive from $X$ another measure, denoted $X^{(n)}$, that is the arithmetic mean of $X$ over $n$ randomly selected distinct participants (thus, $X^{(1)}=X$ ); then one obtains from Eq. 1 the following decomposition:

$X^{(n)}=\mu+\alpha^{(n)}+\beta+\varepsilon^{(n)}$,

where the random variables $\alpha^{(n)}, \beta$, and $\varepsilon^{(n)}$ are always independent with means of zero, but their variances are now $\sigma_{\alpha}^{2} / n, \sigma_{\beta}^{2}$, and $\sigma_{\varepsilon}^{2} / n$, respectively.
Consider now the bivariate distribution of pairs $(x, y)$, where $x$ and $y$ are independent realizations of $X^{(n)}$. Then the population correlation between $x$ and $y$, varying the items, is given by

$\rho(x, y)=\frac{\sigma_{\beta}^{2}}{\sigma_{\beta}^{2}+\sigma_{\varepsilon}^{2} / n}$.

One can recognize in Eq. 3 the expression of a wellknow ICC - that is, the "ICC $(\mathrm{C}, \mathrm{k})$, Cases 2 and $2 \mathrm{~A}$ " coefficient, according to the nomenclature of McGraw and Wong (1996). The expression in Eq. 3 itself shows that the ICC is the proportion of systematic variance $\left(\sigma_{\beta}^{2}\right)$ in the total item-related variance $\left(\sigma_{\beta}^{2}+\sigma_{\varepsilon}^{2} / n\right)$, which also follows from Eqs. 12 and 13 in Courrieu et al. (2011).

Corrected ICC We now consider random variables similar to those defined in Eq. 2, but where a proportion $p$ of values are randomly missing. Then the mean number of averaged values is no longer $n$, but

$n^{\prime}=(1-p) n$.

Denoting $\rho_{p}$ the ICC for variables with a proportion $p$ of randomly missing values, and using Eqs. 3 and 4, one has approximately

$\rho_{p} \approx \frac{\sigma_{\beta}^{2}}{\sigma_{\beta}^{2}+\sigma_{\varepsilon}^{2} / n^{\prime}}$.

So we can write

$$
\begin{gathered}
1 / \rho_{0}-1 / \rho_{p} \approx \frac{\sigma_{\varepsilon}^{2}}{\sigma_{\beta}^{2}}\left(1 / n-1 / n^{\prime}\right) \\
=\frac{n^{\prime}\left(1-\rho_{p}\right)}{\rho_{p}}\left(1 / n-1 / n^{\prime}\right),
\end{gathered}
$$

where $\rho_{0}$ is the exact ICC (without missing values). It follows from the above relation that

$$
\begin{aligned}
\rho_{0} & \approx\left(\frac{n^{\prime}\left(1-\rho_{p}\right)}{\rho_{p}}\left(1 / n-1 / n^{\prime}\right)+\frac{1}{\rho_{p}}\right)^{-1} \\
& =\frac{\rho_{p}}{1-p\left(1-\rho_{p}\right)} \geq \rho_{p},
\end{aligned}
$$

where the last inequality is strict if $\mathrm{p}>0$ and $\rho_{p}<1$, which means that $\rho_{p}$ underestimates $\rho_{0}$. From Eq. 6, one can also define an estimate of $\rho_{0}$ as

$\rho_{\text {cor }}=\frac{\rho_{p}}{1-p\left(1-\rho_{p}\right)}$, 
where the subscript "cor" means "corrected" (for missing data). In practice, $\rho_{p}$ is estimated by the ICC for the data table, computed by a standard ANOVA, and $p$ is the observed proportion of missing data in this table.

One does not obtain $\rho_{c o r}=\rho_{0}$, in general, because randomly missing values induce contaminations between the different sources of variation, in such a way that Eq. 5 is a rough approximation if $\sigma_{\alpha}^{2}$ is not negligible. However, if $\sigma_{\alpha}^{2}$ is small with respect to both $\sigma_{\beta}^{2}$ and $\sigma_{\varepsilon}^{2}$, then $\rho_{c o r}$ appears to be a suitable estimate of $\rho_{0}$. Fortunately, $\sigma_{\alpha}^{2}$ is just the variance corresponding to the column (participant) effect in a data table, and thus this variance is removed when one uses $Z$ scores as behavioral measures. In order to test the suitability of $\rho_{\text {cor }}$ as an estimate of $\rho_{0}$, we built artificial data tables gradually degraded, as in the Adjusted Random Imputation Method section, but instead of considering only $Z$ scores, we used four different versions of each table: a version with noncentered columns (and a substantial $\sigma_{\alpha}^{2}$ ), a version with centered columns (thus, $\sigma_{\alpha}^{2}=0$ ), a $Z$ scores version, and a mixed $Z$ scores version where the $Z$ data in each row were randomly mixed. One can see in Fig. 3 that, when the columns of the data tables are not centered, the estimate $\left(\rho_{c o r}\right)$ has a positive bias that increases as the proportion of missing data increases. However, when the columns are centered, as well as for $Z$ scores and mixed $Z$ scores, the estimate is visibly reliable, since it randomly oscillates in a close neighborhood of the exact value for all proportions of missing data (up to $30 \%$ in these examples, which is much greater than the percentages of missing data commonly observed in real databases). Note that all the plots in Fig. 3 are identical, except the first one. In particular, randomly mixing $Z$ scores in each row of a data table preserves the ICC statistics with respect to the nonmixed case. As a practical rule for the column effect, we observed that $\rho_{\text {cor }}$ is a reliable estimate of $\rho_{0}$ if there is no more than $5 \%$ missing data or if the estimated column effect $\left(s_{\alpha}^{2}\right)$ is not greater than both the row effect $\left(s_{\beta}^{2}\right)$ and the row $\times$ column interaction $\left(s_{\varepsilon}^{2}\right)$. This rule is implemented in the application programs listed in Appendix 1 and Appendix 2.

In summary, we defined a simple and easy-to-compute statistic, denoted $\rho_{\text {cor }}$, which is an ICC corrected for missing data and which is an estimate of the ICC that would be obtained if no data were missing. This estimate is reliable, provided that the column effect in the considered data table is small or removed.

Building virtual participants from data sets with incomplete designs As we observed in the Corrected ICC section, randomly mixing $Z$ scores in each row of a data table preserves the ICC statistics with respect to the nonmixed case. This suggests a possible strategy for structuring data sets with incomplete designs (e.g., the ELP or the FLP), in
Fig. 3 Experiments on artificial data similar to those shown in Fig. 2, but before applying $Z$ score transformations. The "noncentered columns" plot corresponds to raw data, the "centered columns" plot corresponds to the same data with column means of zero, the " $Z$ scores" plot correspond to full $Z$ scores, and the "mixed $Z$-scores" plot corresponds to $Z$ scores randomly mixed in each row. The "exact" curve corresponds to the intraclass correlation coefficient (ICC) of the initial table, the "missing" curve to the ICCs of degraded tables, and the "estimate" curve to the $\rho_{c o r}$ ICCs defined by Eq. 7. Note that all plots, except the first one, are identical
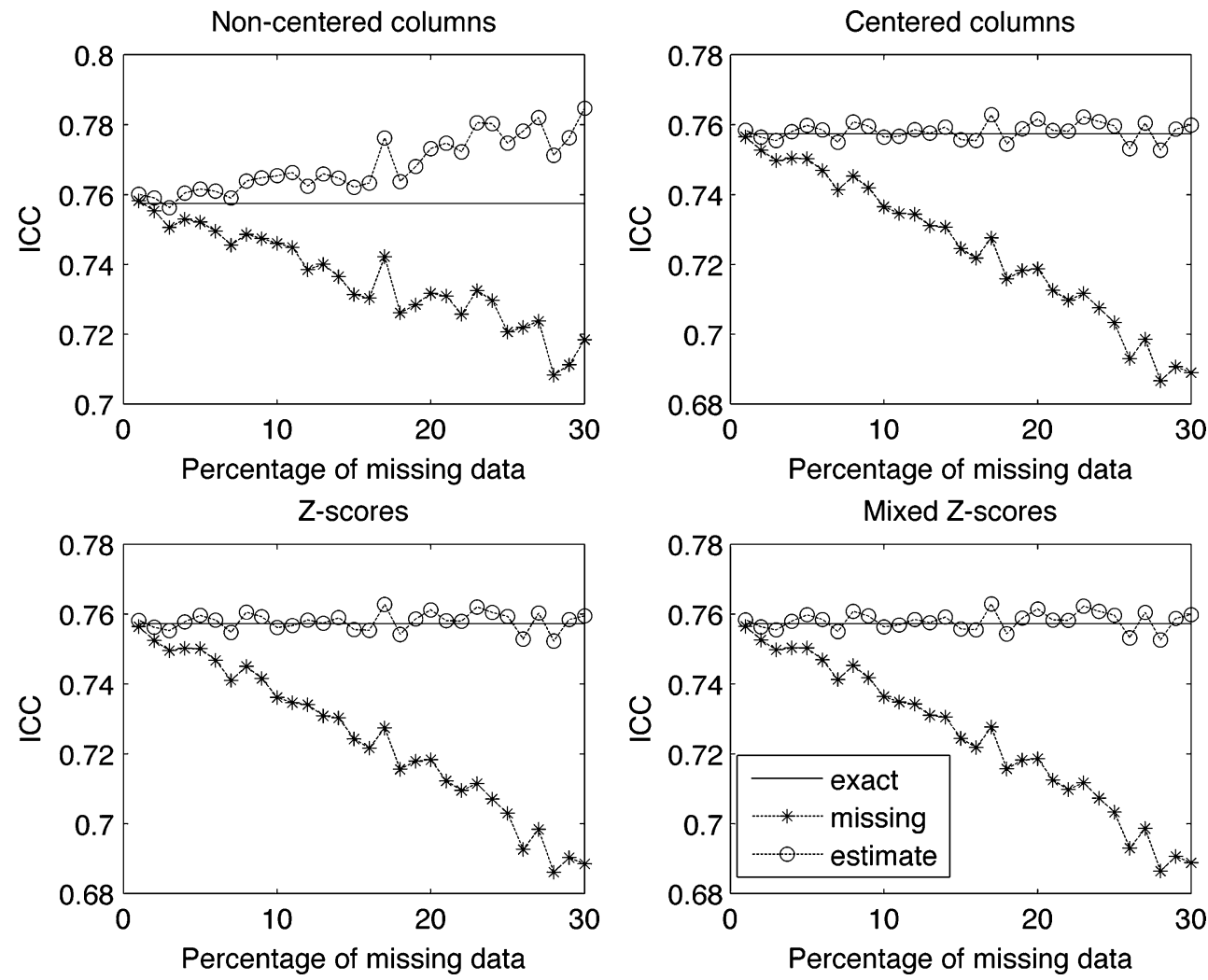
order to make possible the computation of their ICC statistics. First, one must transform the raw data into $Z$ scores in order to remove the linear component of the individual variability. Let $n$ be the observed maximum number of valid data per item; then one can build a rectangular data table with $m$ items (rows) and $n$ virtual participants (columns), and one can randomly assign each valid $Z$ score to a column, in the appropriate row. In general, this leaves "holes" in the table, because there are items with fewer than $n$ valid data. These holes can be treated as ordinary missing data, and the ICC statistics can be computed on the table as if we had $n$ real participants. This approach will not be developed more in this article, and it is mentioned just as an indication for further investigations.

\section{Column and row adjusted random imputation method}

In this section, we define a new imputation algorithm, called column and row adjusted random imputation (CRARI), that allows replacing missing data by imputed values in such a way that the resulting item means are the same as those of the initial data table, and the ICC of the data table under imputation can be set to any desired value in a wide range of possible values, including the ICC of the initial data table (approximately $\rho_{p}$ ), and the estimate $\rho_{\text {cor }}$ of $\rho_{0}$ defined by Eq. 7, when appropriate. In particular, this last option is always suitable for tables of $Z$ scores, as was stated in the Intraclass Correlation Coefficient for Missing Data section. The CRARI algorithm is an extension of the ARI algorithm of Chen et al. (2000); however, it avoids the biases of the ARI method observed in the Adjusted Random Imputation Method section for items $\times$ participants data tables of $Z$ scores.

Principle of the method First, we note that the CRARI algorithm, just like the ARI algorithm, is deterministic whenever there is no more than one missing datum per item in the raw data table. In this case, an imputed value for a given item is necessarily equal to the average of all the valid observations for that item, in order to preserve the item mean. Thus, in the following, we assume that at least one item has more than one missing datum in the raw data table, which is usually the case in large-scale databases.

The first problem to be solved is the case of items with only one valid datum, which leads to imputing values with zero variance when using the ARI method. So the first step of the CRARI method consists in applying the ARI algorithm to each column (instead of each row) of the data table, which results in a provisional imputed data table where all column means are unchanged but each row (item) contains values with a nonzero expected variance, even if only one valid datum is available for that item. At this stage, the item means are not adjusted; that is, they are (probably) different from those of the initial data table. The second step of the CRARI method operates on each row of the provisional data table, where all imputed values (if any) must be centered by subtracting their average. Then one multiplies them by a positive coefficient $c$, whose computation will be described below, and one adds to the imputed values the average of the valid data available for the considered item. As a result, whatever be the coefficient $c$, the item means are now equal to those of the initial data table, leaving also the general mean of the table unchanged. However, the resulting column means can be somewhat different from those of the initial table, despite the fact that their expected value is unchanged. A suitable choice of the coefficient $c$ allows adjusting the ICC of the data table under imputation, while this ICC can be arbitrarily chosen from a wide range of values. Two nonarbitrary ICC values are of special interest: the ICC of the initial data table (hereafter called low ICC), which can always be computed by an ANOVA, and the estimate $\rho_{c o r}$ that can be computed using the low ICC as $\rho_{p}$ in Eq. 7 . Whatever be the target ICC, one can reach it thanks to the following mechanism. Provided that we consider several distinct imputed values for an item, their (nonzero) variance is multiplied by $c^{2}$, and thus, globally, the variance of the data associated to that item increases as $c$ increases or decreases as $c$ decreases. Part of this variance affects the column effect, which is not of interest, but the remaining part of the adjustable variance affects the row $\times$ column interaction effect, which is just what we need to control the ICC, given that the row effect remains unchanged due to the fact that the item means are fixed. As a result, the ratio $q=\sigma_{\beta}^{2} / \sigma_{\varepsilon}^{2}$ decreases as $c$ increases, and conversely, $q$ increases as $c$ decreases. Given that the ICC is a monotonic increasing function of the $q$ ratio, we have a simple mean to control the ICC value of the data table under imputation. So one can use a simple dichotomic search procedure to compute the $c$ value providing the target ICC value, which is the method implemented in the MATLAB program CRARI listed in Appendix 1. This program can be used directly or as an implementation model. For readers not familiar with MATLAB code, we summarize hereafter the method in pseudocode.

CRARI algorithm Given a data table of ( $m$ items) $\times$ ( $n$ participants), with missing data, and a target ICC value ( $\rho_{p}$ computed by ANOVA, or $\rho_{c o r}$ computed from $\rho_{p}$ by Eq. 7): 
If (no more than one missing data per item) then

\{deterministic case $\}$

For item $\leftarrow 1$ to $m$

Replace the missing data, if any, by the average of all valid data in the row.

\section{End}

The output is the resulting table

Else \{random imputation\}

For participant $\leftarrow 1$ to $n$

For each missing data in the column randomly replace the missing data by a valid data from the column.

\section{End}

Compute the average of the replaced data in the column.

Subtract this average from the replaced data in the column.

Compute the average of all valid data in the column.

Add this average to the replaced data in the column.

\section{End}

For item $\leftarrow 1$ to $m$

Compute the average of all replaced data (if any) in the row.

Subtract this average from the replaced data in the row.

\section{End}

$c_{\text {min }} \leftarrow 0, c_{\max } \leftarrow$ maximal $c$ (e.g. 10), tolerance $\leftarrow$ small positive number (e.g. $10^{-4}$ )

\section{Repeat}

$c \leftarrow\left(c_{\min }+c_{\max }\right) / 2$

Compute a provisional table where:

all valid data remain unchanged,

and all replaced data are multiplied by $c$.

Compute the ICC of the provisional table by a standard ANOVA.

If $($ ICC $>$ Target ICC) then

$$
c_{\min } \leftarrow c
$$

\section{Else}

$$
c_{\text {max }} \leftarrow c
$$

\section{End}

Until $\left(c_{\max }-c_{\min }\right)<$ tolerance

The output is the last computed provisional table.

\section{End}


Behavior of the imputation method In order to test the behavior of the new method, we replicated the experiments on artificial data in the Adjusted Random Imputation Method section, but using the CRARI method, instead of the ARI method, for imputation. Figure 4 shows an example of an experiment where the target ICC was the estimate $\rho_{\text {cor }}$. As one can see, the CRARI algorithm reached the target ICC in all cases, providing a close approximation of the exact ICC. Now if one takes the low ICC as the target, one obtains an "imputed" curve indistinguishable from the "missing" curve of Fig. 4. Thus, clearly, the CRARI algorithm is efficient for imputation of data with a prescribed ICC.

In summary, the CRARI method, just like the ARI method, has the advantage of imputing missing data in a way that preserves the original item means. In addition, the CRARI imputation method allows us to preserve the data consistency as measured by the ICC statistics, while one can choose the low ICC observed with missing data or the ICC corrected for missing data $\left(\rho_{c o r}\right)$, as the ICC for the data table under imputation.

\section{ECVT on data tables with imputation of missing data}

A major interest in having an efficient imputation method is to allow the use of permutation resampling techniques that do not work on data tables with too many missing data. This is the case for the ECVT (Courrieu et al., 2011), which was shown to efficiently test the compatibility of any data table with the additive decomposition model (Eq. 1) and

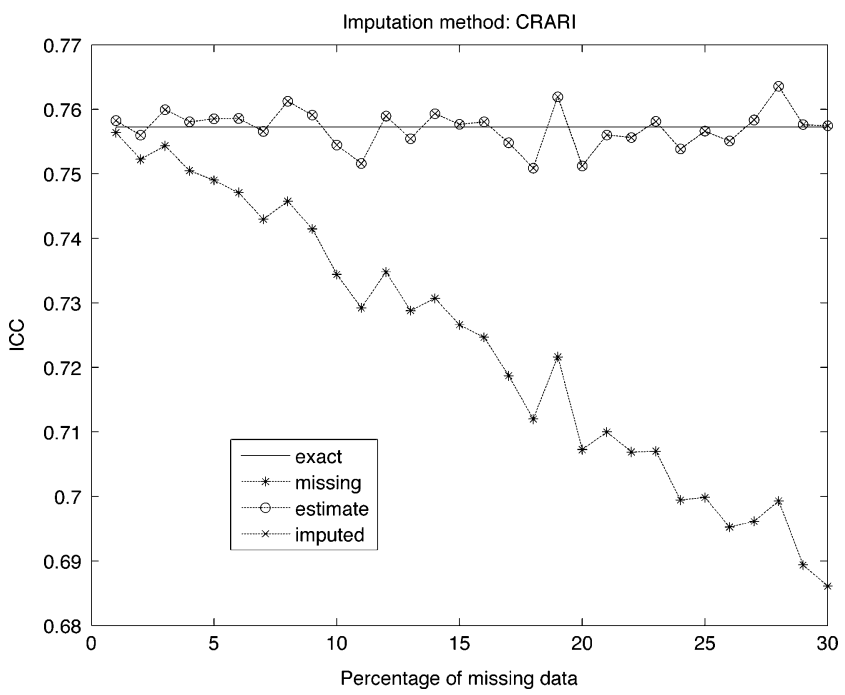

Fig. 4 Similar to Fig. 2, except that the imputation of missing data was performed by the column and row adjusted random imputation (CRARI) method with the $\rho_{c o r}$ intraclass correlation coefficients (ICCs) defined by Eq. 7 as target ICCs consequently, the relevance of the ICC approach for determining the proportion of item-related variance that models should account for in the considered data set. This test cannot work, for instance, on the DLP database (Keuleers et al., 2010), because missing data (16\%) prevent one from obtaining pairs of complete vectors of item means necessary to the permutation resampling procedure of the ECVT. This was, in fact, our first motivation to develop the CRARI method; however, before using it in conjunction with the ECVT, we must be sure that the imputation does not induce biases that could lead the ECVT to provide wrong conclusions.

Building artificial data A suitable way to examine this problem consists of building complete artificial data tables that fulfill or do not fulfill model 1 and degraded versions of the same data tables, including a substantial proportion of missing data, to which one applies the CRARI algorithm in order to impute the missing data. Then, applying the ECVT to both data tables, one must obtain the same conclusions for the tables with imputed data as for the original tables. It is easy to build artificial data that fulfill model 1; however, when building data that do not fulfill model 1, we must take care that the discrepancy of the data from model 1 cannot be removed by the $Z$ score transformation, since the CRARI method is devoted mainly to work on $Z$ scores. One can obtain suitable data using the following generating process:

$x_{i j}=\mu+\operatorname{sign}\left(\beta_{i}\right) \times\left|\beta_{i}\right|^{\alpha_{j}}+\varepsilon_{i j}$,

where $i$ is the index for the row (item) and $j$ is the index for the column (participant). The variables $\mu, \beta$, and $\varepsilon$ are defined as in model 1; however, the participant effect $(\alpha)$ is not additive or multiplicative, since it would be removed by the $Z$ score transformation. The participant effect is a random variable generated by the following process:

$\alpha_{j}=1-s \times \log \left(1-u_{j}\right)$,

where $u_{j}$ is uniformly randomly sampled in the interval $[0,1)$ and $s$ is a nonnegative parameter. Observe that if $s=0$ the participant effect is always equal to 1 , and the generated data fulfill model 1. However, the discrepancy of the generated data from model 1 increases as $s$ increases, as a result of using powers different from 1 in Eq. 8. The cumulative probability function of $\alpha$ in this model is given by

$P(\alpha)=1-\exp (-(\alpha-1) / s), \quad \alpha \geq 1$.

Randomly distributed missing data We randomly generated data tables of size $1,400 \times 80$ using model 8 and varying 
the parameter $s$ of Eq. 9. For each table, we built a degraded version of the table with $16 \%$ randomly missing data, and we transformed the data of both tables into $Z$ scores. Then the CRARI algorithm was applied to the degraded tables, with $\rho_{c o r}$ as the target ICC, resulting in data tables with $16 \%$ imputed data. Finally, we applied the ECVT to both data tables (for a detailed description of this test, see Courrieu et al., 2011). Figure 5 shows an example of ECVT results for data tables generated with $s=0$ (thus, compatible with model 1) and for data tables generated with $s=2$ (thus, incompatible with model 1). As one can see, in all cases, the ECVT provided the correct conclusion, which was always the same for the original data table and for the corresponding imputed data table. Lowering $s$ to 1, one still obtains correct detections of the discrepancy from model 1 for both original and imputed data tables, although the discrepancy is no longer visible on the test graphs. As was previously noted by Courrieu et al. (2011), the ECVT is more sensitive than the eye. We conclude from this study that the ECVT provides similar conclusions for original data tables and for data tables with a substantial proportion of data imputed by the CRARI algorithm, when the missing data are randomly distributed.

Nonrandomly distributed missing data Randomly distributed missing data can occur in certain databases-for instance, when missing data result mainly from technical failures. However, in experimental paradigms such as lexical decision or speeded naming, missing data frequently result from response errors, and these errors are not random, since they typically concentrate on items with low frequency of use and special difficulties (Balota, Cortese, Sergent-Marshall, Spieler, \& Yap, 2004), which are also the items with the greatest RTs (when not missing). Since the DLP database includes lexical decision times, it appeared necessary to verify that the ECVT, combined with the CRARI imputation method, provides suitable conclusions for data tables with a distribution of missing data similar to that of the DLP. In order to do this, we first reordered the rows (items) of the DLP database by increasing order of the item mean RTs transformed into $Z$ scores. Then the exact locations of all missing data in the resulting table were marked. Using Eq. 8, we generated complete artificial data tables of the same size as the DLP data table $(14,056 \times 39)$, varying the $s$ parameter of Eq. 9 from 0 to 6 and using a $q$ ratio approximately equal to that of the DLP $Z$ scores (for $s=0$ ). The rows of each table were reordered by increasing order of their means, and a degraded version of each table was obtained by removing the data at the same locations as the missing data in the reordered DLP data table. Then both tables were transformed into $Z$ scores, and the CRARI
Fig. 5 Expected correlation validity test (ECVT; Courrieu et al., 2011) applied to artificial data that fulfill model 1 , with $s=0$, or that do not fulfill model 1 , with $s=2$. In each case, we tested the original data table and a degraded version with $16 \%$ randomly missing data that were imputed by the column and row adjusted random imputation algorithm with the $\rho_{\text {cor }}$ intraclass correlation coefficient (ICC) defined by Eq. 7 as the target ICC. Note that the ECVT test provided the correct conclusions in all cases, on imputed data tables as well as on original tables
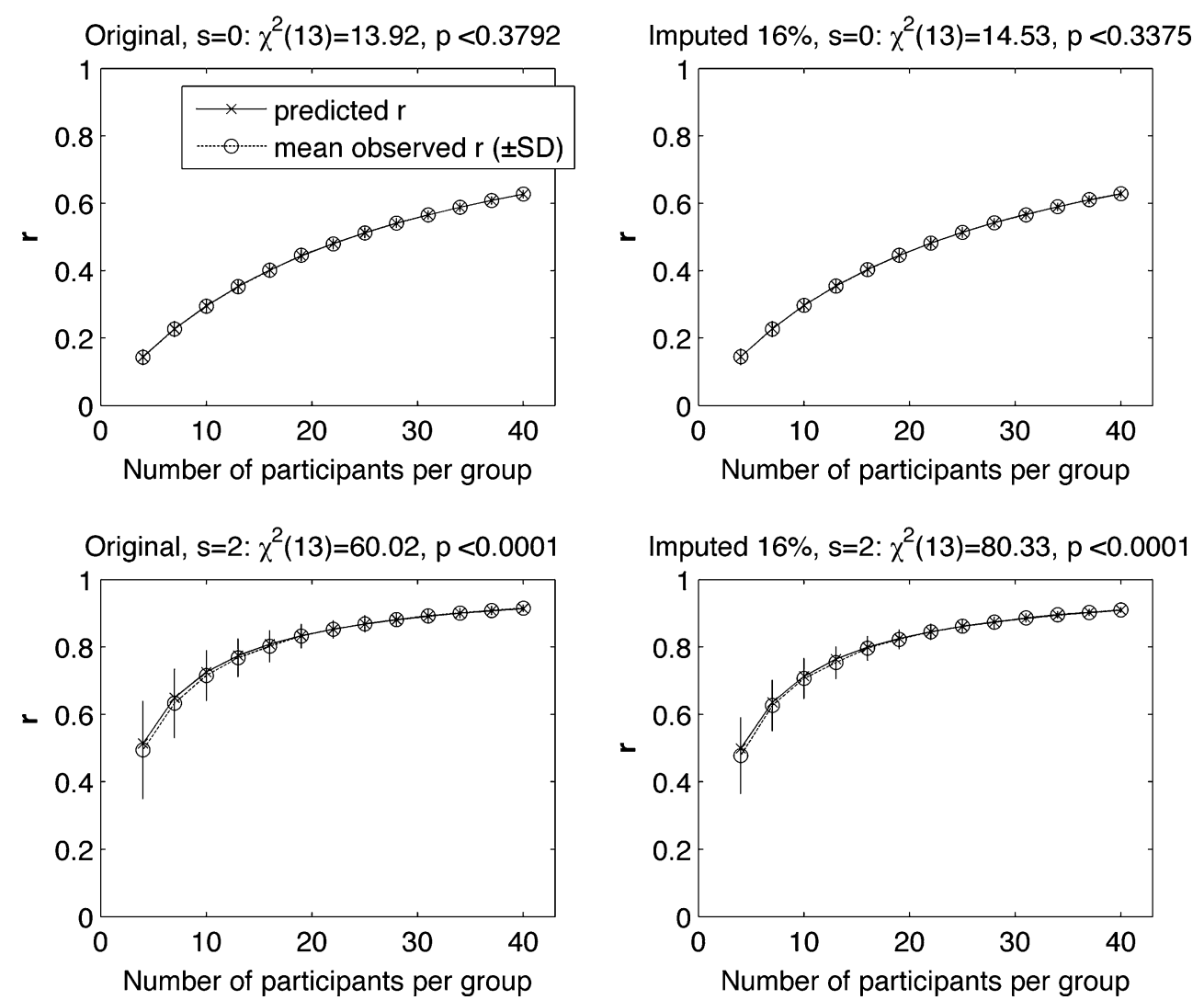
algorithm was applied for imputation of the missing data to the degraded tables, with $\rho_{\text {cor }}$ as the target ICC, and the ECVT was applied to both resulting tables. With such data, it appeared necessary to increase the $s$ parameter of Eq. 9 up to 6 in order to obtain detectable discrepancies from model 1. Outcomes of the ECVT in this experiment are plotted in Fig. 6, where one can see that the conclusions of the ECVT are correct for $s=0$ (compatibility with model 1), as well as for $s=6$ (discrepancy from model 1), for both the original complete data tables and the corresponding data tables under imputation for missing data. However, one can note in Fig. 6 that the standard deviations of the $r$ distributions in the case of discrepancy from model 1 are substantially lower for the data table under imputation than for the original data table, which suggests that the imputation is not unbiased in terms of distribution. Nevertheless, the ECVT seems to provide reliable conclusions, even when combined with the CRARI imputation for missing data.

In summary, we observed that the ECVT provides the same conclusions for data tables without missing data as for similar data tables where a substantial proportion of the data have been replaced with data imputed using the CRARI method. This is true for randomly distributed missing data, as well as for more realistic distributions of missing data that mimic that of the DLP database.

\section{Application to the DLP database}

We computed the ICC and three of its confidence intervals $(95 \%, 99 \%$, and $99.9 \%)$ for four versions of the DLP RT database: the raw data (RTs), the $Z$ scores, the $Z$ scores with imputation of missing data $(16 \%)$ by CRARI with the low ICC, and $Z$ scores with imputation of missing data by CRARI with the $\rho_{\text {cor }}$ ICC. The results are reported in Table 2, where one can see that the statistics, including the confidence intervals, are exactly the same for $Z$ scores and imputed $Z$ scores with low ICC. We note also that using $Z$ scores (imputed or not) improves the reproducible proportion of item-related variance with respect to raw data, and this improvement is significant, since the confidence intervals do not overlap. Note, however, that the last result does not generalize to every database. Depending on each particular data distribution, $Z$ scores are sometimes advantageous, and sometimes they are not, as one can verify using simulated data. The imputation of missing data using $\rho_{\text {cor }}$ clearly provides the most consistent data table. Moreover, if one directly applies Eq. 7 to the confidence limits of the ICC of the DLP $Z$ scores, without imputation for missing data, one obtains the confidence intervals $[0.879,0.884],[0.878$, $0.885]$, and $[0.877,0.886]$ for $95 \%, 99 \%$, and $99.9 \%$ confidence, respectively. These confidence intervals are equal, with three decimal digits, to those obtained with
Fig. 6 Expected correlation validity test (ECVT) in an experiment with artificial data as in Fig. 5, but using the same table size and the same distribution of missing data as the Dutch Lexicon Project database (Keuleers et al., 2010). Once again, the ECVT provided the correct conclusions in all cases, on imputed data tables as well as on original tables
Original, $s=0: \chi^{2}(19)=15.9, p<0.6636$
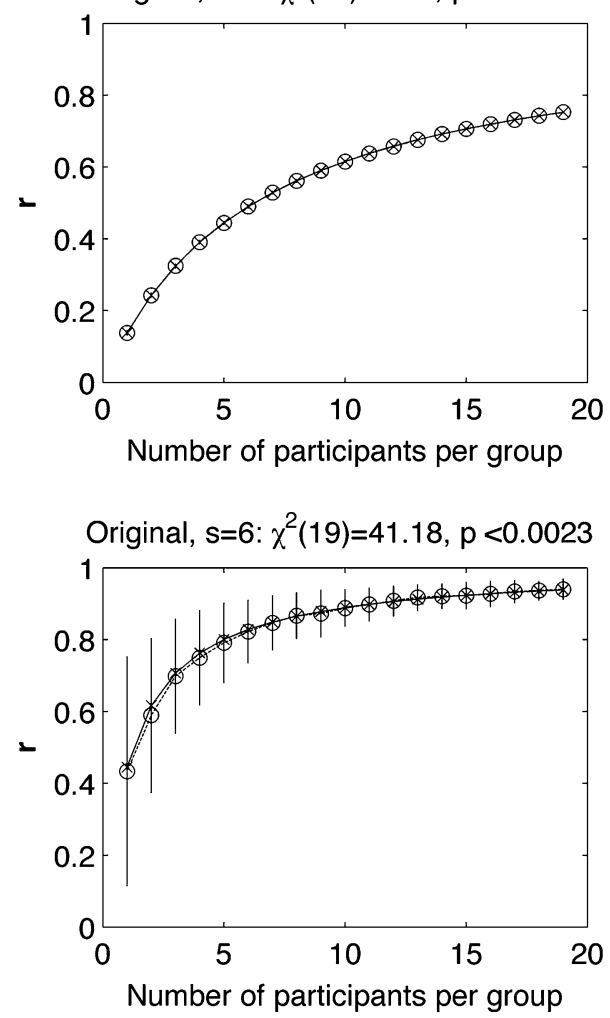

DLP-like imputed, $s=0: \chi^{2}(19)=21.16, p<0.3282$

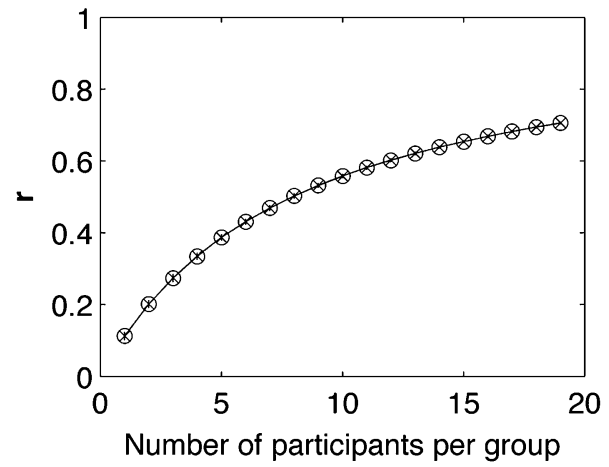

DLP-like imputed, $s=6: \chi^{2}(19)=74.28, p<0.0001$

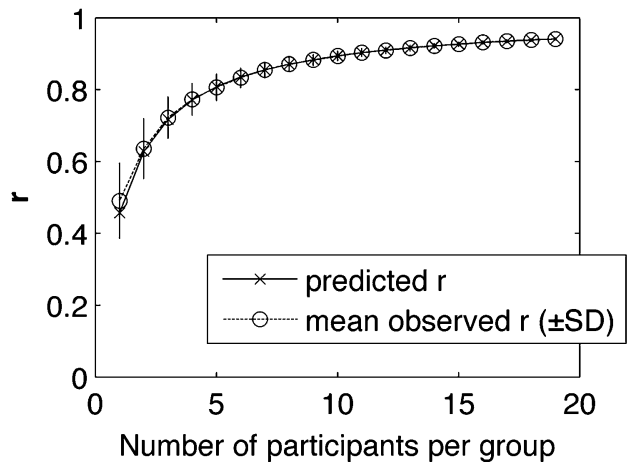


Table 2 Intraclass correlation coefficients (ICCs) with confidence intervals $(95 \%, 99 \%$, and $99.9 \%)$ for four versions of the Dutch Lexicon Project (DLP) response time (RT) database (Keuleers et al., 2010): the raw data (RTs), the $Z$ scores, $Z$ scores with imputation of missing data $(16 \%)$ by the column and row adjusted random imputation (CRARI) method with the low ICC as target, and $Z$ scores with imputation of missing data by CRARI with the $\rho_{\text {cor }}$ ICC as target. The table also provides the estimate of the $q$ ratio $\left(q=\sigma_{\beta}^{2} / \sigma_{\varepsilon}^{2}\right)$ corresponding to each ICC. The $c$ coefficients computed by the CRARI algorithm were $c=2.472$ for the low ICC target and $c=$ 2.1349 for the corrected ICC target

\begin{tabular}{lllll}
\hline DLP Data & ICC $(q$ Ratio $)$ & $95 \%$ Confidence & $99 \%$ Confidence & $99.9 \%$ Confidence \\
\hline Raw data (RTs), 16\% missing & $0.845(0.1396)$ & {$[0.841,0.849]$} & {$[0.840,0.850]$} & {$[0.839,0.851]$} \\
$Z$ scores, 16\% missing & $0.863(0.1610)$ & {$[0.859,0.866]$} & {$[0.858,0.867]$} & {$[0.857,0.868]$} \\
$Z$ scores, low ICC imputation & $0.863(0.1610)$ & {$[0.859,0.866]$} & {$[0.858,0.867]$} & {$[0.857,0.868]$} \\
$Z$ scores, corrected ICC imputation & $0.882(0.1909)$ & {$[0.879,0.884]$} & {$[0.878,0.885]$} & {$[0.877,0.886]$} \\
\hline
\end{tabular}

imputation for missing data with $\rho_{c o r}$ as the target ICC. This provides a rapid way of estimating the confidence intervals of $\rho_{\text {cor }}$ without imputation. At this point, the reader probably asks to what ICC the proportion of variance accounted for by a model must be compared. We break the suspense: Use the raw data ICC if the model correlation is computed with the average raw data, and use the $Z$ scores ICC if the model correlation is computed with the average $Z$ scores. The $\rho_{\text {cor }}$ ICC is useless for testing models (we will see why in the Compensating Item Means Inaccuracy for Model Tests section), unless one uses the $r_{c o r}^{2}$ goodness-of-fit statistic defined in the Corrected $r^{2}$ Goodness-of-Fit Statistic section. However, as was demonstrated in the ECVT on Data Tables With Imputation of Missing Data section, it is relevant to impute missing data using $\rho_{\text {cor }}$ as the target ICC, in order to be able to test properties of the data themselves.

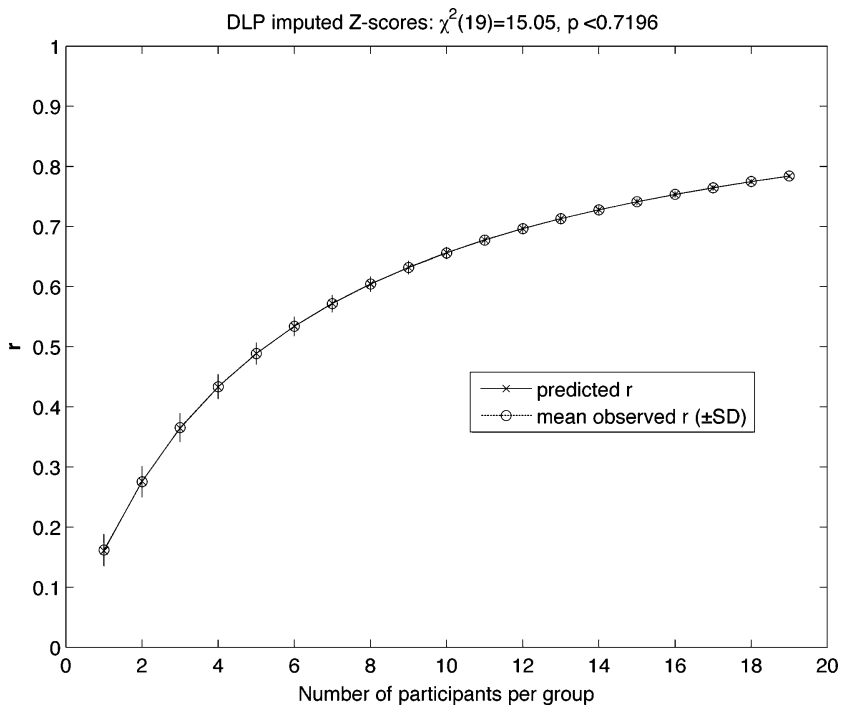

Fig. 7 Expected correlation validity test of the Dutch Lexicon Project (DLP) database (Keuleers et al., 2010), after $Z$ score transformation of the data, and imputation of the $16 \%$ missing data by the column and row adjusted random imputation algorithm with the $\rho_{\text {cor }}$ intraclass correlation coefficient (ICC) defined by Eq. 7 as the target ICC. The "predicted" and "observed" curves are indistinguishable, and the $\chi^{2}$ test is clearly nonsignificant, so the $Z$ scores of the DLP database fulfill model 1 , and the ICC approach is relevant for these data
This is just what we do now, applying the ECVT to the DLP $Z$ scores with CRARI imputation of missing data, using $\rho_{\text {cor }}$ as the target ICC. The result of the ECVT is shown in Fig. 7, where one can see that the theoretical and observed correlation curves are indistinguishable and the $\chi^{2}$ test is nonsignificant, indicating that the DLP data are compatible with model 1.

As a supplemental verification, we applied the ECVT to the DLP $Z$ scores with CRARI imputation of missing data, but using the low ICC as the target. The result of the ECVT is shown in Fig. 8, where one can see that, once again, the theoretical and observed correlation curves are indistinguishable and the $\chi^{2}$ test is nonsignificant, confirming that the DLP data are compatible with model 1. So, the statistics reported in Table 2 can be confidently used to test models with the DLP database.

The validity of the ICC approach had been previously demonstrated, using the ECVT, for word identification times in English and for word-naming times in English and

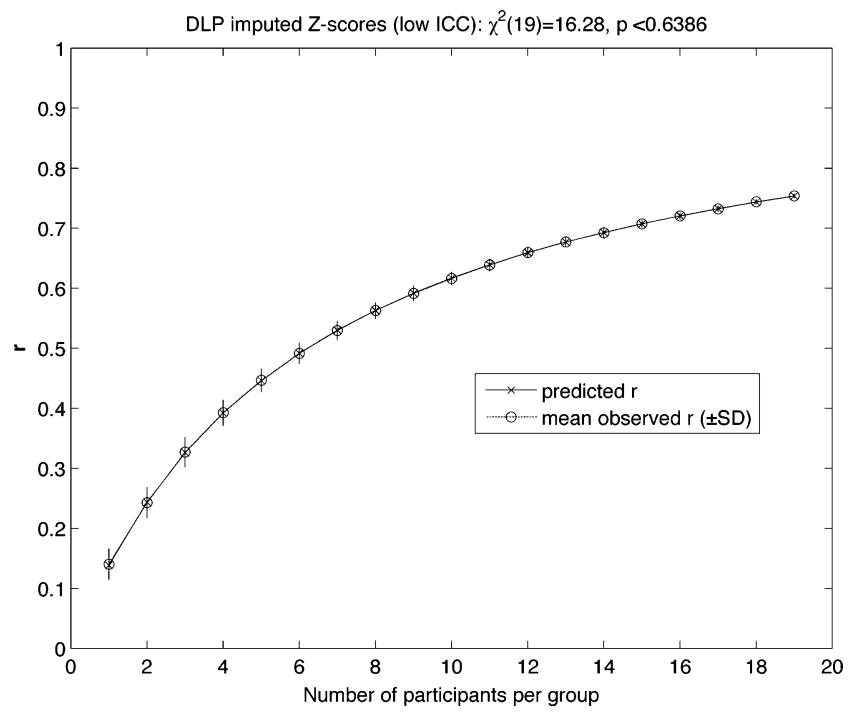

Fig. 8 Similar to Fig. 7, but using the low intraclass correlation coefficient (ICC) as the target ICC of the column and row adjusted random imputation for missing data. The conclusion of the expected correlation validity test is unchanged. DLP, Dutch lexicon project 
in French (Courrieu et al., 2011). However, this is the first time this can be concluded with regard to lexical decision times (in Dutch), which is very important since lexical decision is the most widely used experimental paradigm in visual word recognition studies. An alternative approach would be to remove all items presenting a high proportion of missing data and to apply the ECVT only on the remaining items, without imputation for missing data. This approach leads to removing a large proportion of items with low frequency of use, which is not desirable because rare words are useful in experiments, where their relative frequency of occurrence is much greater than in everyday life. Moreover, statistics computed only on the most frequent items are not necessarily good estimates for the whole item population.

In summary, we provided the ICC statistics (with confidence intervals) for the lexical decision times of the DLP database and their $Z$ score transformation. Using the CRARI imputation method allowed us to apply the ECVT to DLP $Z$ scores and to conclude that the ICC suitably measures the reproducible proportion of item-related variance that models should try to account for in the $Z$ score version of the DLP database.

\section{Compensating item means inaccuracy for model tests}

Missing data induce inaccuracy in item means It is well known that the empirical mean of a random sample of size $n$ is an unbiased, consistent estimator of the parent mean. More precisely, as $n$ increases, the estimation error of the mean rapidly tends to be normally distributed with a mean of 0 and variance $\left(E\left(v^{2}\right)-E^{2}(v)\right) / n$, where $v$ is the considered random variable and $E$ is the usual expected value operator. Thus, preserving the empirical item means in imputation methods is certainly a good strategy, because empirical means are unbiased estimators of the parent means; however, the accuracy of the estimates clearly depends on the number of empirical data actually averaged for each item, while this number is affected by missing data. As a result, the accuracy of the empirical vector of item means decreases as the proportion of missing data increases in the data table. This is illustrated in Fig. 9, were the results of an experiment with artificial data similar to those in the Adjusted Random Imputation Method and the Column and Row Adjusted Random Imputation Method sections are plotted, together with the correlation between the vector of item means of the original data table and the one of degraded data tables with an increasing proportion of missing data (up to $90 \%$ ). As one can see, the degradation of the correlation of item means [" $r$ (item means)" curve] is roughly parallel to the degradation of the low ICC ("missing" curve). At the same time, the $\rho_{\text {cor }}$ ICC ("estimate" curve) and the CRARI imputation ICC

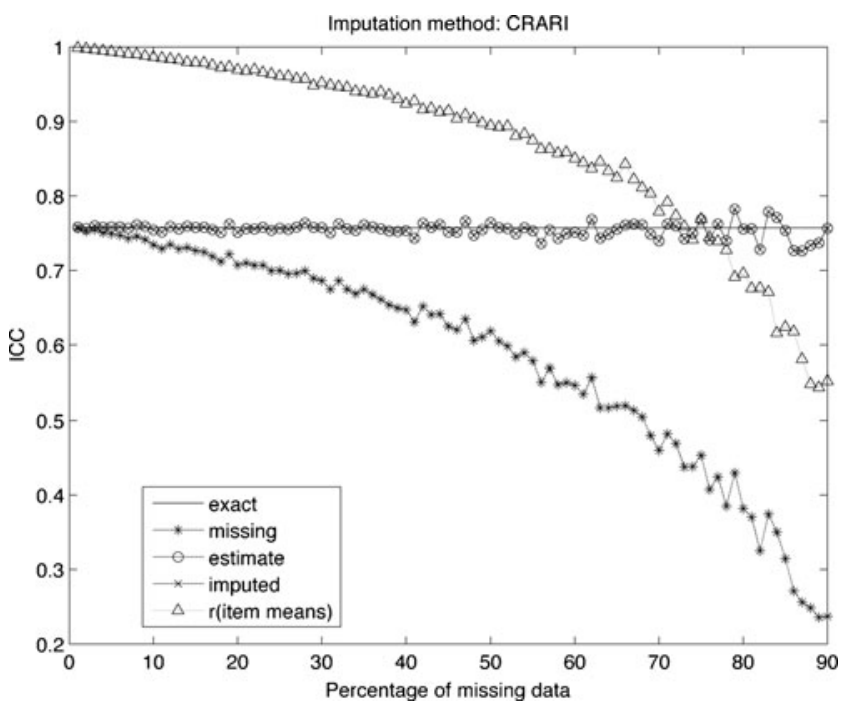

Fig. 9 An experiment on artificial data similar to the experiment of Fig. 4, but with degraded data tables up to $90 \%$ missing data, and the evolution of the correlation between the original item means and the item means of degraded data tables. CRARI, colum and row adjusted random imputation; ICC, intraclass correlation

("imputed" curve) remain equal and oscillate in a neighborhood of the exact ICC ("exact" curve), with a moderately increasing variance. This is the reason why the low ICC is usually the good reference to test models, since one uses the (squared) correlation between model predictions and possibly degraded empirical item means, according to Courrieu et al. (2011).

Virtues of the $r^{2}$ /ICC ratio However, there is another way of testing predictor type models, and that way is, in a sense, more general than the simple model misfit test proposed in Courrieu et al. (2011). Noting that the usual squared Pearson's correlation coefficient $r^{2}$ (or $R^{2}$, for multiple regression models) is an estimate of the proportion of itemrelated variance accounted for by a given model, we deduce that the ratio $r^{2} / I C C$ estimates the proportion of reproducible item-related variance that is accounted for by the model, given that the ICC estimates the reproducible proportion of item-related variance actually available in the data. But there is more, as we state now.

The parent ratio of $r^{2} / I C C$ is $\rho^{2}(x, B) / \rho(x, y)$, where $B$ is the considered predictive variable (model prediction) and $x$ and $y$ are two independent realizations of $X^{(n)}$, as in Eq. 3 . Remembering that $\operatorname{Var}(x)=\operatorname{Var}(y)=\sigma_{\beta}^{2}+\sigma_{\varepsilon}^{2} / n$ (see Courrieu et al., 2011), one can write

$$
\begin{aligned}
\rho^{2}(x, B) / \rho(x, y) & =\frac{\operatorname{Cov}^{2}(x, B)}{\operatorname{Var}(x) \operatorname{Var}(B)} / \frac{\operatorname{Cov}(x, y)}{(\operatorname{Var}(x) \operatorname{Var}(y))^{1 / 2}} \\
& =\frac{\operatorname{Cov}^{2}(x, B)}{\operatorname{Var}(B) \operatorname{Cov}(x, y)},
\end{aligned}
$$


and given that

$$
\begin{aligned}
\operatorname{Cov}(x, B) & =\operatorname{Cov}(\beta, B)+\operatorname{Cov}\left(\varepsilon^{(n)}, B\right), \operatorname{Cov}(x, y) \\
& =\sigma_{\beta}^{2}, \text { and } \operatorname{Var}(B)=\sigma_{B}^{2},
\end{aligned}
$$

one obtains

$\rho^{2}(x, B) / \rho(x, y)=\frac{\left(\operatorname{Cov}(\beta, B)+\operatorname{Cov}\left(\varepsilon^{(n)}, B\right)\right)^{2}}{\sigma_{\beta}^{2} \sigma_{B}^{2}}$.

$$
\text { If } \operatorname{Cov}\left(\varepsilon^{(n)}, B\right)=0 \text {, then }
$$

$\rho^{2}(x, B) / \rho(x, y)=\frac{\operatorname{Cov}^{2}(\beta, B)}{\sigma_{\beta}^{2} \sigma_{B}^{2}}=\rho^{2}(\beta, B)$,

which is just the squared correlation between the predictive variable $B$ and the hidden behavioral variable $\beta$. Note that this quantity is independent of the noise and of the number of participants, which makes it of special interest for various purposes and, in particular, for removing the effect of missing data from model goodness-of-fit statistics. The case of Eq. 12 is what normally occurs if the predictive variable $B$ does not overfit the data.

However, if $\operatorname{Cov}(\beta, B) \operatorname{Cov}\left(\varepsilon^{(n)}, B\right)>0$, then Eq. 11 implies that $\rho^{2}(x, B) / \rho(x, y)>\rho^{2}(\beta, B)$. This case typically corresponds to an overfitting; that is, the predictive variable $B$ is correlated with the data noise (with the same sign as its correlation with $\beta$ ), generally due to the use of too many free parameters in the model to fit the data.

Now, if $\operatorname{Cov}(\beta, B) \operatorname{Cov}\left(\varepsilon^{(n)}, B\right)<0$, then Eq. 11 implies that $\rho^{2}(x, B) / \rho(x, y)<\rho^{2}(\beta, B)$; however, one can hardly imagine a mechanism generating such a situation (negative overfitting?), except hazard.

In order to illustrate Eq. 12, we considered two behavioral databases previously used in Courrieu et al. (2011), and in Rey, Brand-d'Abrescia, Peereman, Spieler, and Courrieu (2010). These databases are an English wordnaming RT table of size 770 items $\times 94$ participants, with $3.61 \%$ missing data, and a French word-naming RT table of size 615 items $\times 100$ participants, with $3.94 \%$ missing data. For each database, we considered two well-known predictors - that is, the word log-frequency of use and the word length (number of letters). We used a permutation resampling procedure with various participant group sizes, similar to the procedure used in the ECVT. However, in addition to the computation of the correlation between item means for two groups, at each resampling step, we also computed the correlation between item means for one group and each of the two predictors. At the end, we obtained, for each participant group size, an estimate of the ICC and estimates of the correlations between item means and the two predictors. Then, for each group size and for each predictor, we computed the ratio $r^{2} / I C C$, and we plotted the obtained ratios as functions of the participant group size, shown in Fig. 10, for the two databases. We know that the ICC is a monotonic increasing function of the number of participants; however, Eq. 12 predicts that $r^{2}$ increases in the same way as the ICC, in such a way that the ratio $r^{2} / I C C$ remains constant. This is just what we can observe in Fig. 10 for the two databases and the two predictors. Incidentally, we can also observe that the word frequency effect is stronger than the word length effect in English, while the converse is true in French.

Corrected $r^{2}$ goodness-of-fit statistic Here, we exploit the fact that Eq. 12 is independent of the noise and of the number of participants. Let $r_{p}$ be the correlation coefficient of a predictive variable $B$ with a behavioral measure, averaged over $n$ participants with a proportion $p$ of missing values, and let $\rho_{p}$ denote the corresponding ICC. If there is no overfitting, then one has, after Eq. 12,

$r_{p}^{2} / \rho_{p}=r^{2}(\beta, B)$,

and, in particular for $p=0$ one has

$r_{0}^{2} / \rho_{0}=r^{2}(\beta, B) \Rightarrow r_{0}^{2}=\rho_{0} r^{2}(\beta, B)$.

Combining Eqs. 13 and 14, one obtains

$r_{0}^{2}=\rho_{0} r_{p}^{2} / \rho_{p}$.

Now, in cases where $\rho_{c o r}$ is a suitable estimate of $\rho_{0}$ (e.g., $Z$-score-type data), we have

$r_{0}^{2} \approx \rho_{c o r} r_{p}^{2} / \rho_{p}$

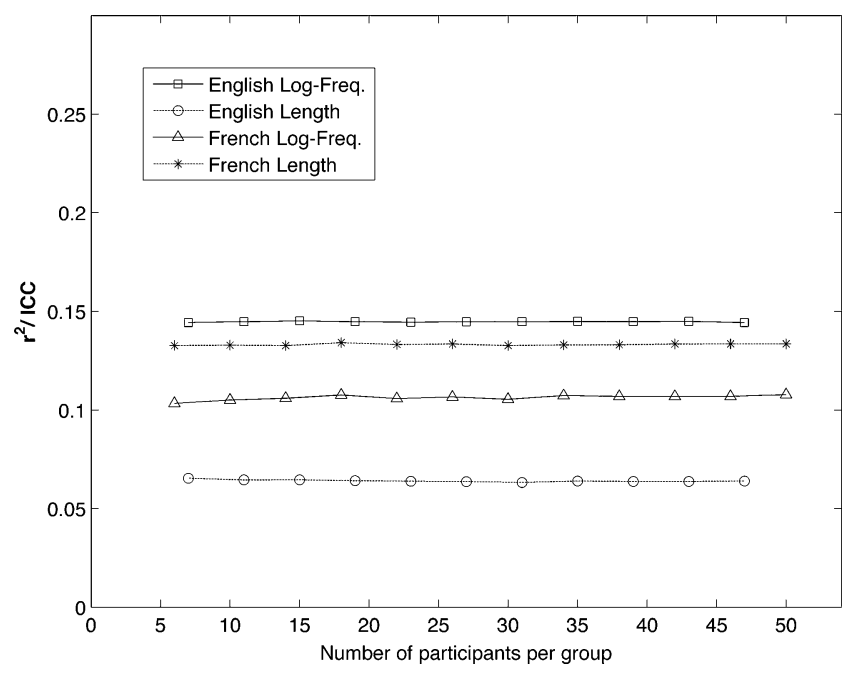

Fig. 10 Evolution of the $r^{2} / I C C$ ratio as a function of the number of participants taken into account, for two predictors (word logfrequency of use and word length in letters) of word-naming times in two languages (English and French), using two empirical databases from Courrieu et al. (2011). ICC, intraclass correlation coefficient 
that is, multiplying the observed ratio $r^{2} /$ ICC by the ICC corrected for missing data ( $\rho_{\text {cor }}$ defined by Eq. 7), we obtain an estimate of the squared correlation of the predictive variable $B$ with the behavioral measure averaged over $n$ participants without missing data. So, using Eq. 16, we define the $r^{2}$ statistic corrected for missing data as

$r_{c o r}^{2}=\rho_{c o r} r_{p}^{2} / \rho_{p}$.

Note that the reference ICC for the goodness-of-fit statistic $r_{c o r}^{2}$ is $\rho_{\text {cor }}$, whose confidence intervals can be computed on a data table under CRARI imputation with $\rho_{\text {cor }}$ as the target ICC or can be directly approximated using Eq. 7 on the ICC confidence limits. The MATLAB program ICCR2 listed in Appendix 2 takes as arguments a data table and a set of predictors, and it provides as outputs the ICC statistics of the data table and the goodness-of-fit statistics of the predictors $\left(r^{2}, r^{2} / I C C\right.$, and $\left.r_{c o r}^{2}\right)$. An example of use of the program is also provided. The goodness-of-fit statistics can also be used as complementary information in multiple regression analyses, remembering that $R^{2}$ is equal to the squared correlation coefficient of the data with the optimal composite predictor computed by multiple regression analysis (Cohen, Cohen, West, \& Aiken, 2003).

In order to illustrate the behavior of the $r_{c o r}^{2}$ estimate, we built artificial data tables as in the Missing Data Induce Inaccuracy in Item Means section and an artificial predictor with known $r^{2}$ goodness of fit. Then we increased the proportion of missing data up to $90 \%$, and we plotted $r_{0}^{2}$ ("exact" curve), $r_{p}^{2}$ ("observed" curve), and $r_{c o r}^{2}$ ("estimate" curve) as functions of the percentage of missing data, shown in Fig. 11. As one can see, $r_{c o r}^{2}$ behaves as a nearly unbiased estimator of $r_{0}^{2}$; however, the estimator variance increases as the proportion of missing data increases. Nevertheless, the estimator variance remains small enough for practical use with commonly observed percentages of missing data. Thus, we can conclude that $r_{c o r}^{2}$ is a suitable estimate for practical applications, while the bias of the observed $r_{p}^{2}$ is visibly substantial and dramatically increasing as the proportion of missing data increases. So we can be sure that $r_{c o r}^{2}$ is at least a much better estimate than the usual $r^{2}$ in cases in which there are missing data, provided that one uses $Z$-score-type data.

In summary, after observing that missing data degrade the accuracy of the averages used as item performance measures, in the same way as they degrade the data consistency, we proposed two useful goodness-of-fit statistics to test models: the ratio $r^{2} / I C C$, which is approximately independent of data accuracy and consistency, and the $r_{c o r}^{2}$ statistic, which is a version of the usual $r^{2}$ statistic corrected for missing data. The $r_{c o r}^{2}$ statistic can be compared with $\rho_{c o r}$ in the same way as the usual $r^{2}$ statistic can be compared with the low ICC.

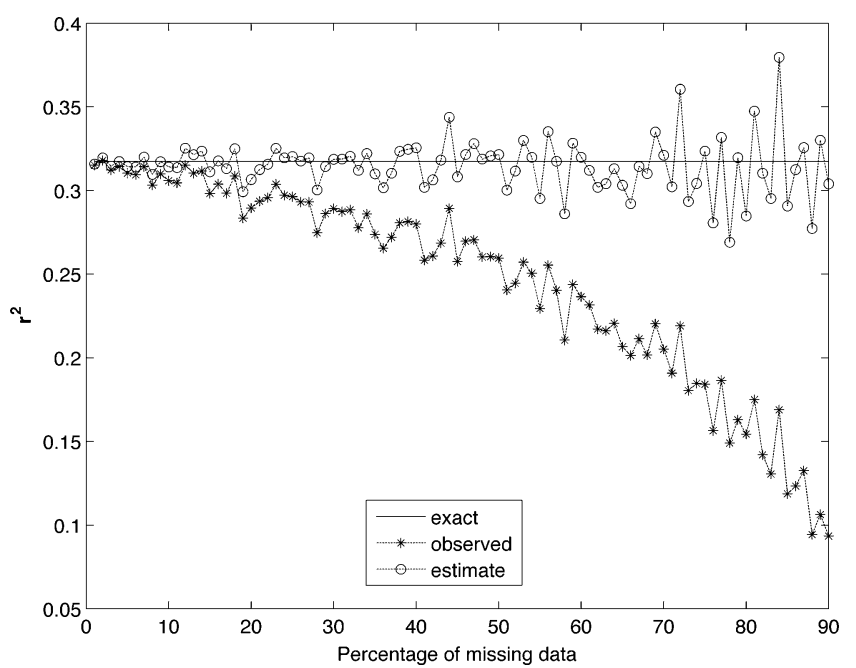

Fig. 11 An experiment using artificial data similar to those in Fig. 9 and an artificial predictor with known $r^{2}$ with the original data. The figure plots the observed $r^{2}$ goodness-of-fit statistics and the $r_{c o r}^{2}$ estimates as functions of the percentage of missing data in the degraded data tables

\section{Conclusion}

This study is a continuation of the work recently published by Courrieu et al. (2011), which addressed the problem of the amount of variance that item-level performance models should account for in large-scale behavioral databases. In the previous study, it was shown that the reproducible proportion of item-related variance in such databases is suitably measured by a particular ICC computed on the data table, provided that the considered behavioral measure fulfills a usual additive decomposition model. Then a powerful test, named ECVT, was proposed to detect discrepancies of the considered data from this model, and it was shown that commonly used behavioral measures suitably fulfill the model, making the ICC approach relevant. However, the ECVT is based on a permutation resampling procedure that cannot correctly work when there are too many missing data in the considered data table. Unfortunately, the proportion of missing data is commonly quite large in large-scale databases, due to response errors, outliers, and technical failures. So, in the present study, we addressed the problem of missing data, and we proposed ways of overcoming this problem. We noted that the $Z$ score transformation of raw data (Faust et al., 1999) is a general solution for suitably formatting large-scale behavioral databases, so the present study focused mainly on $Z$-score-type data.

The first idea that one can have to solve the problem of missing data is to replace the missing data with suitable estimates that preserve the essential statistical properties of the whole data set, without introducing biases. We first 
described the ARI method of Chen et al. (2000), and using artificial data, we observed some drawbacks of this method for an application to large-scale item-level behavioral databases. Then we showed that the ICC of data tables with missing data is biased, and we described a suitable estimate of the exact ICC of any Z-score-type data table with missing data. We described a new missing data imputation method, called CRARI, which is a suitable extension of the ARI method that allows adjusting the data ICC. A MATLAB program implementing the CRARI method is listed in Appendix 1, together with an example of application to real data.

Then we demonstrated, using artificial data, that the ECVT proposed by Courrieu et al. (2011) provides the same results on data tables without missing data as on similar data tables where a substantial proportion of data has been removed and imputed by the CRARI method. We applied the CRARI imputation method to the DLP database (Keuleers et al., 2010), which then allowed us to apply the ECVT to this database under imputation of missing data and to conclude that the ICC approach is relevant for this database. This is the first time that the approach of Courrieu et al. has been validated for lexical decision times, which is an important result, since lexical decision is the most widely used experimental paradigm in visual word recognition studies. The ICC statistics of the DLP database were provided for practical use in model testing on these data.

However, we observed that missing data not only degrade the data table ICC, but also degrade the average values used as item performance measures. So we proposed corrected correlation statistics suitable to solve this problem when one tests models and predictors on data sets with missing data. A MATLAB program computing these statistics is listed in Appendix 2, together with an example of application to real data. Note that users having difficulties using the provided MATLAB programs can directly contact the authors (pierre. courrieu@univ-provence.fr).

We conclude that this study provides effective and reasonably robust methods for solving the problem of missing data that always occurs in large-scale studies and for testing item performance models and predictors on the large-scale behavioral databases recently made available to the community of researchers.

Acknowledgments The authors would like to thank the anonymous reviewers and the action editor, Dr. Ira Bernstein, for their helpful comments. Part of this study was funded by ERC Research Grant 230313. 
Appendix 1 CRARI program (MATLAB 7.5 code) for missing data imputation. Texts to the right of "\%" are comments

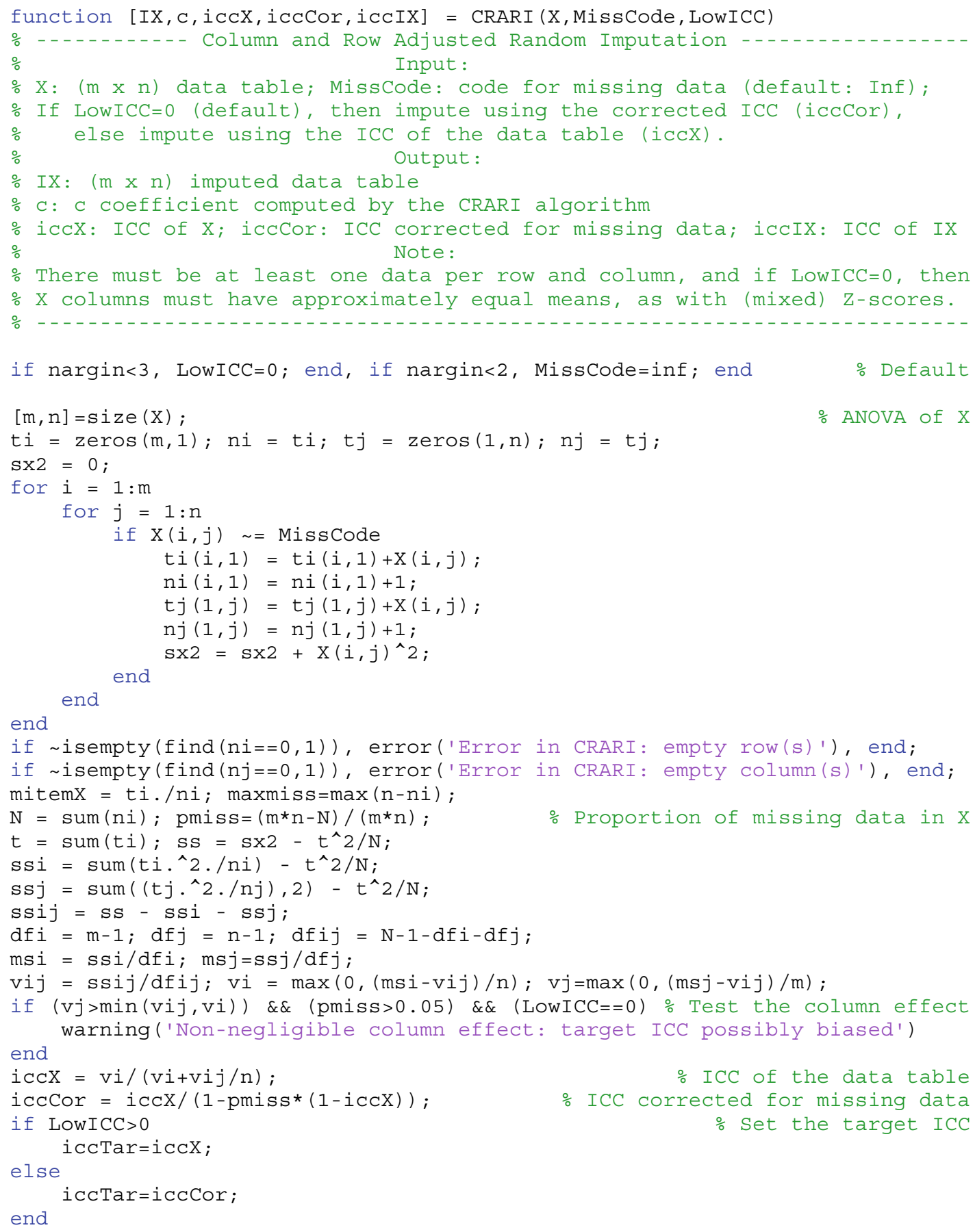

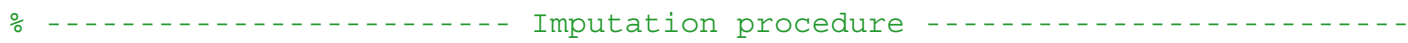
if pmiss>eps

$I X=\operatorname{zeros}(m, n)$;

if maxmiss<2 을 Deterministic imputation case

for $i=1: m$

$\mathrm{x}=\mathrm{X}(i,:) ; \quad i \mathrm{x}=\mathrm{IX}(i,:)$;

miss $=f$ ind $(x==$ MissCode) ; 


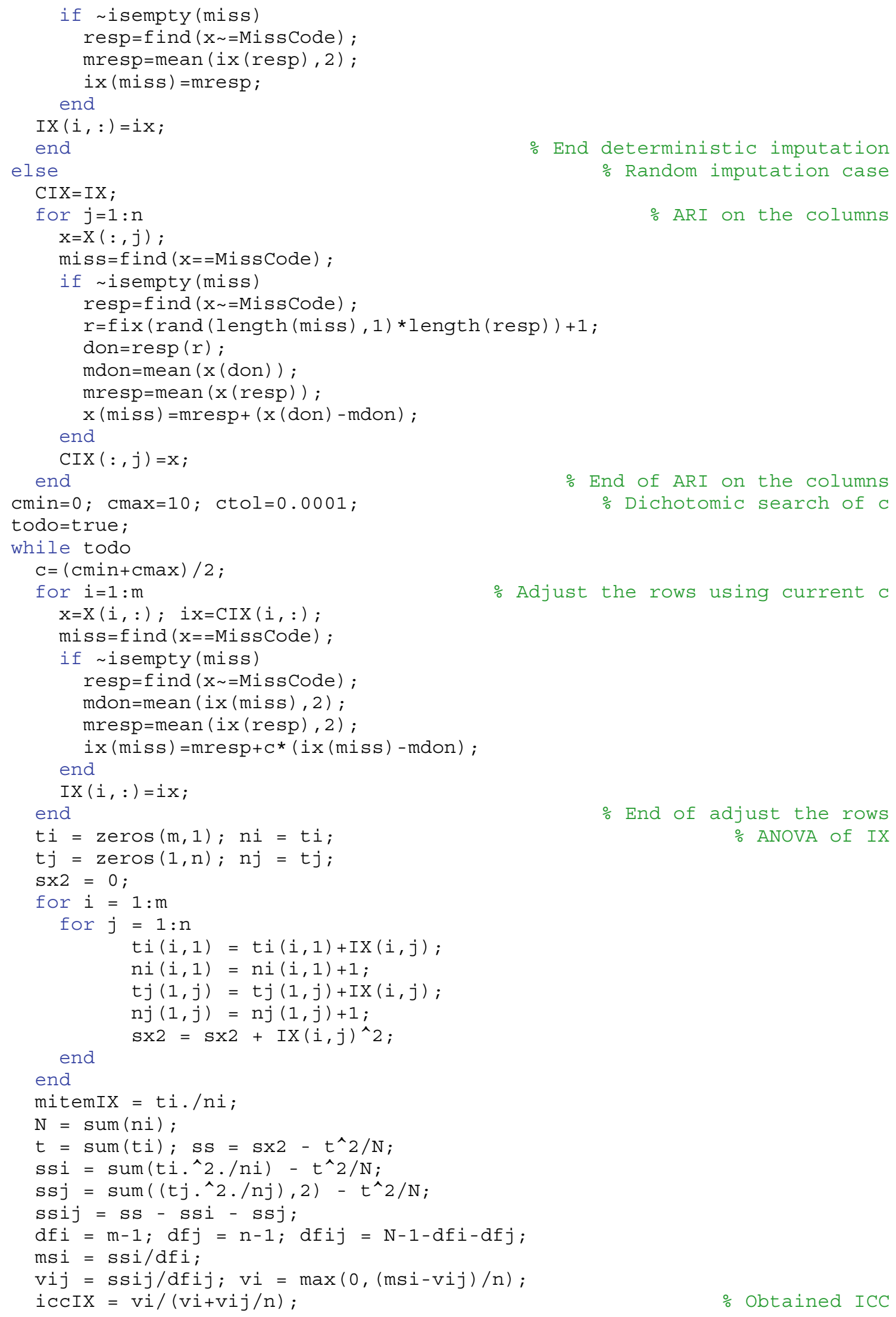

\% End deterministic imputation \% Random imputation case

ARI on the columns

\% Adjust the rows using current $\mathrm{C}$

\% End of ARI on the columns \% Dichotomic search of $\mathrm{C}$ 


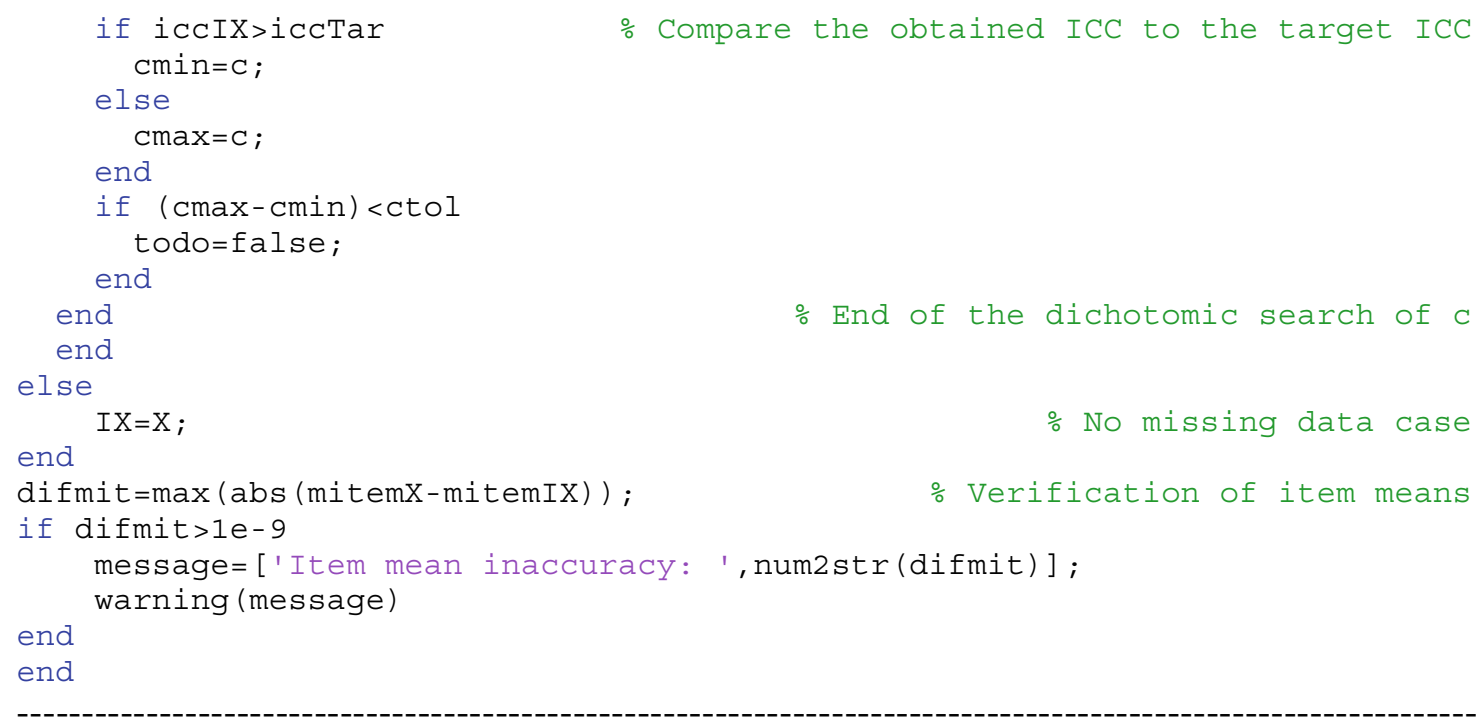

Commented example of use of the CRARI program

$>$ load DLP.mat

$\%$ Load DLP raw data $(R T s)$

$>>$ ZDLP=zscore $(\mathrm{DLP}, 0)$;

$\%$ Transform DLP raw data in Z-scores (the argument " 0 " is the code for missing data in DLP, but the code for missing data in ZDLP is "inf", since 0 is a possible Z-score value)

$>[\mathrm{m}, \mathrm{n}]=\operatorname{size}(\mathrm{ZDLP}) ; \mathrm{pmiss}=\operatorname{sum}(\operatorname{sum}(\mathrm{ZDLP}==\mathrm{inf})) /(\mathrm{m} * \mathrm{n})$

pmiss $=0.1568$

$\%$ This is the proportion of missing data in ZDLP (and in DLP)

>> [IZDLP,cIZDLP,iccZDLP,iccCorZDLP,iccIZDLP] = CRARI(ZDLP,inf,0);

\% CRARI imputation of the missing data in ZDLP, using ZDLP ICC corrected for missing data (iccCorZDLP) as target ICC (i.e. setting LowICC $=0$ as third input argument). If one set LowICC =1 as third input argument, then the target ICC is the observed one (iccZDLP).

>> cIZDLP,iccZDLP,iccCorZDLP,iccIZDLP

cIZDLP $=2.1349 \quad \%$ Obtained $c$ coefficient

iccZDLP $=0.8626 \quad \%$ ICC of the input data table $(Z D L P)$

iccCorZDLP $=0.8816 \quad \%$ ICC corrected for missing data

iccIZDLP $=0.8816 \quad \%$ ICC of the output data table $(I Z D L P)$ 


\section{Appendix 2 ICCR2 program (MATLAB 7.5 code). Texts at right of "\%" are comments}

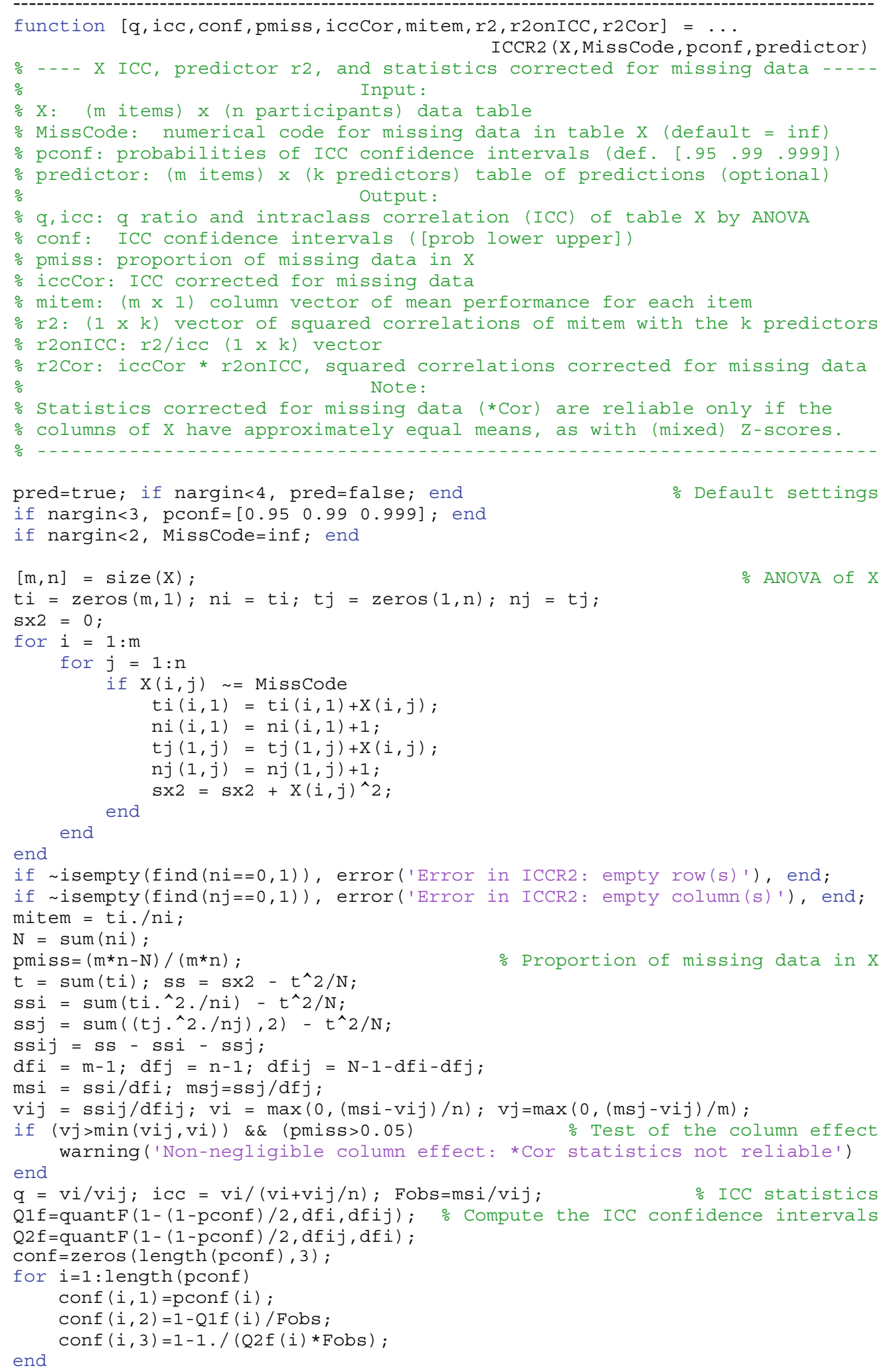




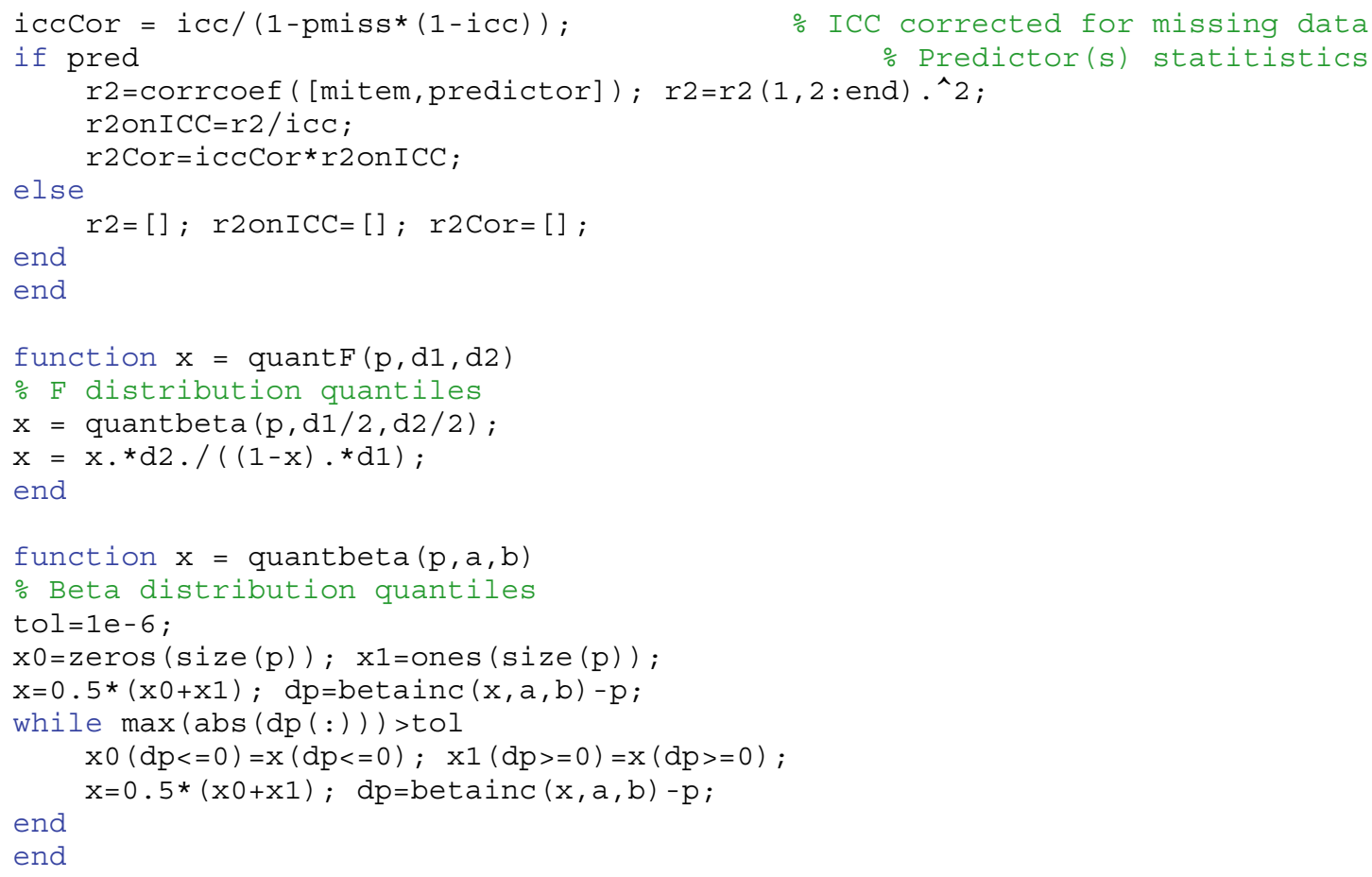

end

Commented example of use of the ICCR2 program

$>>$ [q, icc, conf, pmiss, iccCor, mitem, r2, r2onICC, r2Cor $]=\ldots$

ICCR2(EnglishRT, 0, 0.99, [EnglishLogFreq, EnglishLength]);

$\%$ The "EnglishRT" input argument is a (770 $x$ 94) table of English printed word naming times (raw data from Courrieu et al., 2011) with 3.61\% missing data, "0" is the code for missing data, we request a 99\% confidence interval for the ICC, and we test two predictors: word log-frequency of use (EnglishLogFreq), and word length in letters (EnglishLength).

$>$ q, icc, conf, pmiss, iccCor

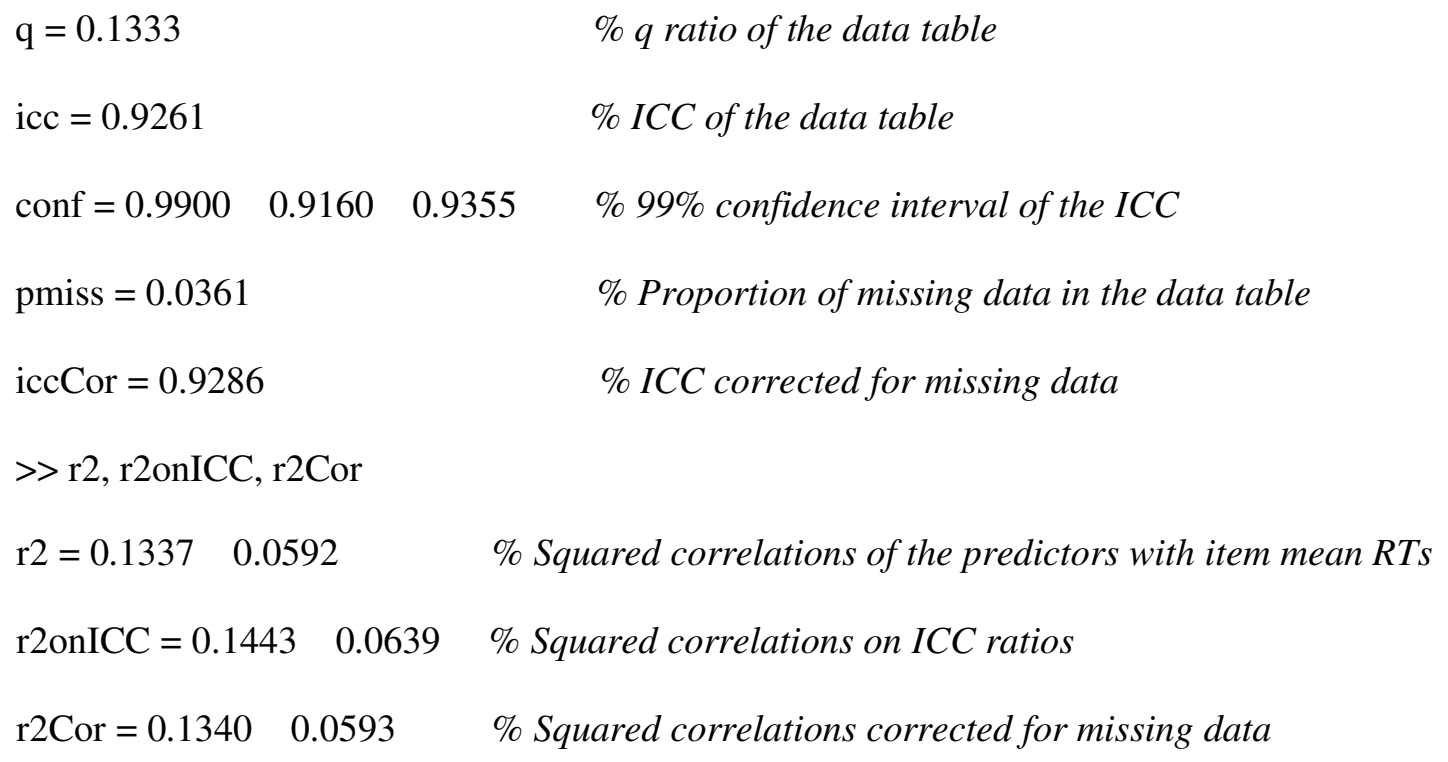




\section{References}

Balota, D. A., Cortese, M. J., Sergent-Marshall, S. D., Spieler, D. H., \& Yap, M. J. (2004). Visual word recognition of single-syllable words. Journal of Experimental Psychology: General, 133, 283-316.

Balota, D. A., Yap, M. J., Cortese, M. J., Hutchison, K. A., Kessler, B., Loftis, B., et al. (2007). The English Lexicon Project. Behavior Research Methods, 39, 445-459.

Chen, J., Rao, J. N. K., \& Sitter, R. R. (2000). Efficient random imputation for missing data in complex surveys. Statistica Sinica, 10, 1153-1169.

Cohen, J., Cohen, P., West, S. G., \& Aiken, L. S. (2003). Applied multiple regression/correlation analysis for the behavioral sciences (3rd ed.). London: Erlbaum.

Courrieu, P., Brand-D’Abrescia, M., Peereman, R., Spieler, D., \& Rey, A. (2011). Validated intraclass correlation statistics to test item performance models. Behavior Research Methods, 43, 37-55. doi:10.3758/s13428-010-0020-5

Faust, M. E., Balota, D. A., Spieler, D. H., \& Ferraro, F. R. (1999). Individual differences in information-processing rate and amount: Implications for group differences in response latency. Psychological Bulletin, 125, 777-799.

Ferrand, L., New, B., Brysbaert, M., Keuleers, E., Bonin, P., Méot, A., et al. (2010). The French Lexicon Project: Lexical decision data for 38,840 French words and 38,840 pseudowords. Behavior Research Methods, 42, 488-496.

Keuleers, E., Diependaele, K., \& Brysbaert, M. (2010). Practice effects in large-scale visual word recognition studies: A lexical decision study on 14,000 Dutch mono- and disyllabic words and nonwords. Frontiers in Psychology, 1, 174. doi:10.3389/fpsyg. 2010.00174

McGraw, K. O., \& Wong, S. P. (1996). Forming inferences about some intraclass correlation coefficients. Psychological Methods, 1, 30-46.

Perry, C., Ziegler, J. C., \& Zorzi, M. (2010). Beyond single syllables: Large-scale modeling of reading aloud with the Connectionist Dual Process (CDP++) model. Cognitive Psychology, 61, 106151. doi:10.1016/j.cogpsych.2010.04.001

Rey, A., Brand-d'Abrescia, M., Peereman, R., Spieler, D., \& Courrieu, P. (2010, November). The nanopsycholinguistic approach: Item performance in disyllabic word naming. Paper presented at the 51st Annual Meeting of the Psychonomic Society, St Louis.

Rey, A., \& Courrieu, P. (2010). Accounting for item variance in largescale databases. Frontiers in Psychology, 1, 200. doi:10.3389/ fpsyg.2010.00200

Rey, A., Courrieu, P., Schmidt-Weigand, F., \& Jacobs, A. M. (2009). Item performance in visual word recognition. Psychonomic Bulletin \& Review, 16, 600-608.

Yap, M. J., \& Balota, D. A. (2009). Visual word recognition of multisyllabic words. Journal of Memory and Language, 60, 502-529. 